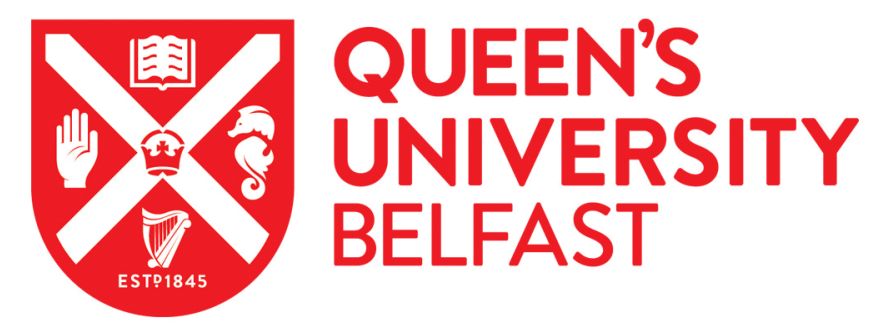

\title{
An Experimental Evaluation of Switched Combining Based Macro- Diversity for Wearable Communications Operating in an Outdoor
} Environment

Yoo, S. K., Cotton, S. L., Scanlon, W. G., \& Conway, G. A. (2017). An Experimental Evaluation of Switched Combining Based Macro-Diversity for Wearable Communications Operating in an Outdoor Environment. IEEE Transactions on Wireless Communications, 16(8), 5338-5352. https://doi.org/10.1109/TWC.2017.2709298

Published in:

IEEE Transactions on Wireless Communications

Document Version:

Publisher's PDF, also known as Version of record

Queen's University Belfast - Research Portal:

Link to publication record in Queen's University Belfast Research Portal

Publisher rights

() Copyright 2017 IEEE. This is an open access article published under a Creative Commons Attribution License

(https://creativecommons.org/licenses/by/4.0/), which permits unrestricted use, distribution and reproduction in any medium, provided the author and source are cited.

\section{General rights}

Copyright for the publications made accessible via the Queen's University Belfast Research Portal is retained by the author(s) and / or other copyright owners and it is a condition of accessing these publications that users recognise and abide by the legal requirements associated with these rights.

Take down policy

The Research Portal is Queen's institutional repository that provides access to Queen's research output. Every effort has been made to ensure that content in the Research Portal does not infringe any person's rights, or applicable UK laws. If you discover content in the

Research Portal that you believe breaches copyright or violates any law, please contact openaccess@qub.ac.uk. 


\title{
An Experimental Evaluation of Switched Combining Based Macro-Diversity for Wearable Communications Operating in an Outdoor Environment
}

\author{
Seong Ki Yoo, Student Member, IEEE, Simon L. Cotton, Senior Member, IEEE \\ William G. Scanlon, Senior Member, IEEE and Gareth A. Conway, Member, IEEE
}

\begin{abstract}
This paper investigates the potential improvement in signal reliability for outdoor wearable communications channels operating at $868 \mathrm{MHz}$ using switched combining based macrodiversity. In this study, a number of different macro-diversity configurations consisting of two and four base stations were considered to help mitigate the impact of body shadowing upon a wearable node which was located on the central chest region of an adult male. During the field measurements, five different walking movements were performed and then analyzed to investigate the efficacy of using macro-diversity. It was found that all of the considered switched combining schemes including switch-and-stay combining (SSC), switch-and-examine combining (SEC) and SEC with post-examining selection (SECps) provided a worthwhile signal improvement when an appropriate switching threshold was adopted. The maximum diversity gain obtained in this study was found to be $19.5 \mathrm{~dB}$ when using four-base station SECps. The diversity gain, the number of path examinations and the number of path switches between base stations for the switched combiner output varied according to the determined switching threshold, highlighting the importance of the selection of an appropriate threshold level. Furthermore, the performance/complexity trade off is demonstrated. Finally, the fading behavior at the output of the switched diversity combiners was then characterized using the diversity specific equations developed under the assumption of independent and non-identically distributed Nakagami- $m$ fading channels. Over all of the measurement scenarios considered in this study, the theoretical models provided an adequate fit to the fading observed at the output of the virtual switched combiner.
\end{abstract}

Index Terms-diversity gain, macro-diversity, Nakagami- $m$ fading, shadowing, switched combining, wearable communications.

\section{INTRODUCTION}

$\mathbf{C}$ Ombining the signal obtained from spatially separated antennas is one well-known method of mitigating the detrimental effects of fading and improving the signal reliability and the performance of wireless communication systems [1], [2]. Since spatial diversity schemes generally require no additional transmit power or bandwidth, they are more commonly applied compared to other diversity techniques such as time, frequency and polarization diversity [3]. Spatial diversity schemes are often implemented using two main configurations, namely micro- and macro-diversity which are differentiated

S. K. Yoo, S. L. Cotton, W. G. Scanlon and G. A. Conway are with the Institute of Electronics, Communications and Information Technology, Queen's University Belfast, U.K., BT3 9DT (e-mail: \{syoo02, simon.cotton, w.scanlon, g.conway\}@qub.ac.uk). according to the allocation of the antennas. In micro-diversity based systems, the antennas are typically positioned within a single base station and their spacing is in the order of, or shorter than the carrier wavelength. The expectation here is that the antennas will experience different small-scale fading (multipath). On the other hand, in macro-diversity based systems, the antennas are located in a number of different spatially separated base stations and their spacing is much longer than the carrier wavelength. This causes the antennas to experience different large-scale fading (shadowing). Hence, micro- and macro-diversity are generally employed to combat the effects of multipath fading and shadowing, respectively [4].

Switched combining and gain combining are the most prevalent combining schemes utilized in conjunction with microand macro-diversity based systems [5]. In switched combining schemes, the receiver selects one of the available diversity paths according to a corresponding criterion. This category of combiners contains both the pure selection combining (PSC) and threshold selection combining (TSC) techniques. On the other hand, in gain combining schemes, the output of combiner is formed as a linear combination of the signals received by all of the diversity paths. This class of combiner includes the equal gain combining (EGC) and maximum ratio combining (MRC) techniques. In general, a better performance can be achieved by gain combining schemes compared to switched combining schemes. Furthermore, it is widely recognized that MRC is generally considered as the optimal combining scheme in terms of the performance. Nevertheless, switched combining has remained popular due to its relatively low complexity and ease of implementation. In a PSC system, the combiner requires continuous and simultaneous knowledge of all of the possible signal branches, which can be both time- and powerconsuming. Contrastingly, in a TSC system, the receiver stays with the current branch as long as its signal-to-noise ratio (SNR) is above the predetermined switching threshold. In other words, the receiver switches from the current branch to another only when its SNR falls below the predetermined switching threshold [6]. Therefore, this approach prevents the unnecessary monitoring of the SNR of all branches but more importantly avoids needless switching between branches especially when the SNR of the currently selected branch is at level which is sufficient to provide the desired information recovering capacity. The TSC grouping of combiners consists 
mainly of the switch-and-stay combining (SSC), switch-andexamine combining (SEC) and SEC with post-examining selection (SECps) schemes.

Over the last few decades, a number of studies investigating spatial diversity techniques have been performed in the context of wearable or equivalently body-centric communications [714]. However, the majority of these have considered only PSC, EGC and MRC schemes [7-12]. As mentioned above, these combining schemes can be resource consuming compared to simpler TSC schemes and therefore may be impractical for implementation in wearable systems which favor low complexity, low cost and ultra-low power architectures [15], [16]. To this end, the authors of [13], [14] have studied cooperative diversity using the SEC scheme for on-body communications channels. In [13], it was shown that a cooperative switched combining scheme provided an improvement in outage probability (OP), a reduction in power consumption and a low switching rate. In [14], the author demonstrated that the outage performance of the cooperative switched combining scheme was not considerably degraded, but it had a much lower switching rate between branches when compared to the traditional cooperative PSC scheme.

Moreover, the majority of the studies on spatial diversity techniques for wearable communications which have been presented in the literature have focused on micro-diversity systems positioned either on the human body or at a base station with the aim of mitigating the impact of multipath fading [7-9]. However, the use of micro-diversity alone may not be sufficient to overcome the arguably more critical signal degradations caused by random shadowing events induced by the human body and obstacles in the local surroundings. To overcome human body shadowing in wearable communications channels, spatial diversity using antennas distributed across the human body has been proposed [17], [18]. For example, in [17], front and back positioned antennas used together with a PSC scheme provided mitigation of human body shadowing and an overall improvement in signal reliability in outdoor body-to-body communications channels at $2.45 \mathrm{GHz}$. In [18], six receiver antennas were distributed across the front and back torso of the human body to help overcome the detrimental effects of human body shadowing in off-body communications within an indoor environment at $868 \mathrm{MHz}$. The benefit of having more than two signal branches was demonstrated by comparing the diversity gains between dualbranch and six-branch receiver configurations. Nonetheless, there are many drawbacks to constructing diversity systems designed to be worn on the human body, especially when compared to integrating the technology into a local base station or using combining opportunities offered by multiple base stations. These include potential obtrusion to the user, the additional weight added to the wearable system, associated circuity and enclosures and also the extra drain on battery life.

To the best of our knowledge, a systematic investigation of the utilization of TSC based macro-diversity to overcome shadowing for outdoor wearable communications has yet to appear in the open literature. Therefore, in this paper, we examine the potential improvement in signal reliability for outdoor wearable communications channels operating at $868 \mathrm{MHz}$ using the switched combined signal forwarded from multiple spatially distributed base stations. It is widely known that an SSC scheme gains no further benefit from having more than two signal branches [19]. Accordingly, we consider dual-base station SSC, $L$-base station SEC and $L$-base station SECps schemes in this study. Most importantly though, for the first time, we statistically characterize the fading behavior observed at the output of the switched diversity combiners, i.e., from the perspective of combiner output, using the diversity specific equations which were developed under the assumption of independent and non-identically distributed (i.n.i.d.) Nakagami- $m$ fading channels.

The remainder of the paper is organized as follows. In Section II, we briefly review the statistical characteristics of the Nakagami- $m$ fading model before introducing theoretical equations for the cumulative distribution functions ( $\mathrm{CDFs})$ and probability density functions (PDFs) of $L$-base station SSC, SEC and SECps schemes operating in i.n.i.d. Nakagami$m$ fading channels. In Section III, we describe the measurement set-up, experiments and environments. In Section IV, the achievable diversity gain, correlation, number of path examinations and number of path switches for the SSC, SEC and SECps schemes are presented and compared with those for the PSC scheme. Model fitting for the SSC, SEC and SECps schemes is presented in Section V while a further performance analysis is conducted while considering the OP for the three schemes in Section VI. Finally, Section VII concludes the paper with some closing remarks.

\section{First-Order Statistics of Switched Combiners OPERATING NAKAGAMI- $m$ FADING ENVIRONMENTS}

\section{A. The Nakagami-m Fading Model}

The Nakagami- $m$ fading model has been proven to characterize the fading behavior of a diverse range of wireless channels such as those found in body-centric [20], [21] and land-mobile [22], [23] communications systems. It is a general statistical model which includes as special cases a number of well-known distributions such as the one-sided Gaussian $(m=0.5)$ and Rayleigh $(m=1)$ distributions [24]. It can also be used to approximate the Rician distribution when $m>1$. As well as modeling small scale fading, the Nakagami$m$ model can be used to describe shadowing of the signal amplitude owing to its relationship with the gamma distribution. It is already well established that the gamma distribution can be used to model shadowing of the signal power [25], [26] and since gamma and Nakagami- $m$ random variables are related by a simple quadratic transformation, accordingly the signal power or equivalently SNR over a Nakagami- $m$ fading channel is distributed according to the gamma distribution [6], [27]. The PDF, $f_{\gamma}(\gamma)$, and CDF, $F_{\gamma}(\gamma)$ of the SNR over the Nakagami- $m$ fading channels can be expressed as [6]

$$
\begin{gathered}
f_{\gamma}(\gamma)=\frac{m^{m} \gamma^{m-1}}{\Gamma(m) \bar{\gamma}^{m}} \exp \left(-\frac{m \gamma}{\bar{\gamma}}\right) \\
F_{\gamma}(\gamma)=1-\frac{\Gamma\left(m, \frac{m \gamma}{\bar{\gamma}}\right)}{\Gamma(m)}
\end{gathered}
$$


where $m$ is the fading severity parameter, $\bar{\gamma}=E[\gamma]$ is the average SNR where $E[\cdot]$ is the expectation operator, $\Gamma(\cdot)$ is the gamma function and $\Gamma(\cdot, \cdot)$ is the upper incomplete gamma function [28].

\section{B. L-base Station SSC operating over Nakagami-m Fading Channels}

For the SSC scheme, when the received SNR at the currently selected branch, or equivalently in this case base station, falls below the predetermined switching threshold the receiver simply switches from one base station to another base station and then stays with that base station irrespective of its channel condition. When the fading observed at each of the base stations is assumed to be i.n.i.d. the CDF and PDF of the output SNR at an $L$-base station SSC combiner can be written as follows [19]

$$
\begin{aligned}
& \sum_{i=0}^{L-1}\left(\sum_{j=0}^{L-1} \frac{1}{F_{j}\left(\gamma_{T}\right)}\right)^{-1} F_{i}(\gamma), \quad \gamma<\gamma_{T} \\
& F_{\mathrm{SSC}}(\gamma)=\left\{\begin{array}{l}
\sum_{i=0}^{L-1} \lambda_{i}\left[F_{i}(\gamma)-F_{j}\left(\gamma_{T}\right)\right] \\
\quad+\left(\sum_{j=0}^{L-1} \frac{1}{F_{j}\left(\gamma_{T}\right)}\right)^{-1} F_{i}(\gamma), \quad \gamma \geq \gamma_{T}
\end{array}\right. \\
& f_{\mathrm{SSC}}(\gamma)=\left\{\begin{array}{cc}
\sum_{i=0}^{L-1}\left(\sum_{j=0}^{L-1} \frac{1}{F_{j}\left(\gamma_{T}\right)}\right)^{-1} f_{i}(\gamma), & \gamma<\gamma_{T} \\
\sum_{i=0}^{L-1}\left[\lambda_{i}+\left(\sum_{j=0}^{L-1} \frac{1}{F_{j}\left(\gamma_{T}\right)}\right)^{-1}\right] f_{i}(\gamma), & \gamma \geq \gamma_{T}
\end{array}\right.
\end{aligned}
$$

where $L$ is the number of base stations, $\gamma_{T}$ is the fixed switching threshold, $f_{i}(\cdot)$ and $F_{i}(\cdot)$ denote PDF and CDF for $i^{\text {th }}$ base station, respectively, and $\lambda_{i}$ is the probability that the $i^{\text {th }}$ base station is used in the SSC scheme, which can be obtained using an $L$-state Markov chain such that

$$
\lambda_{i}=\left(\sum_{j=0}^{L-1} \frac{1}{F_{j}\left(\gamma_{T}\right)}\right)^{-1} \frac{1}{F_{i}\left(\gamma_{T}\right)}, i=0,1, \ldots, L-1 .
$$

Now substituting (1) and (2) into (4), we can obtain the PDF of the output SNR of an $L$-base station SSC combiner operating over i.n.i.d. Nakagami- $m$ channels as given in (6).

\section{L-base station SEC operating over Nakagami-m Fading Channels}

For the SEC scheme, when the received SNR at the currently selected base station falls below the predetermined switching threshold the receiver switches from the current base station to another base station and examines its SNR.
If it is not above the predetermined switching threshold, the receiver switches to another base station and examines its SNR again. The receiver repeats this process until either it finds an acceptable base station which is above the switching threshold or determines that all base stations are not acceptable. In the latter case, it usually uses the last examined base station. Again, for i.n.i.d. fading at each of the base stations, the CDF and PDF of the output SNR at an $L$-base station SEC combiner can be expressed as [19]

$$
F_{\mathrm{SEC}}(\gamma)= \begin{cases}\sum_{i=0}^{L-1} \lambda_{i} F_{i}(\gamma)\left[\prod_{k=0, k \neq i}^{L-1} F_{k}\left(\gamma_{T}\right)\right], & \gamma<\gamma_{T} \\ \sum_{i=0}^{L-1} \lambda_{i} \prod_{k=0}^{L-1} F_{k}\left(\gamma_{T}\right)+\sum_{i=0}^{L-1} \sum_{j=0}^{L-1} \lambda_{(i-j)_{L}} & \\ \times\left[F_{i}(\gamma)-F_{i}\left(\gamma_{T}\right)\right] \prod_{k=0}^{j-1} F_{(i-j+k)_{L}}\left(\gamma_{T}\right), & \gamma \geq \gamma_{T}\end{cases}
$$

$$
f_{\mathrm{SEC}}(\gamma)= \begin{cases}\sum_{i=0}^{L-1} \lambda_{i}\left[\prod_{k=0, k \neq i}^{L-1} F_{k}\left(\gamma_{T}\right)\right] f_{i}(\gamma), & \gamma<\gamma_{T} \\ \sum_{i=0}^{L-1}\left[\sum_{j=0}^{L-1} \lambda_{(i-j)_{L}} \prod_{k=0}^{j-1} F_{(i-j+k)_{L}}\left(\gamma_{T}\right)\right] f_{i}(\gamma), \gamma \geq \gamma_{T}\end{cases}
$$

where $(a)_{b}$ denotes $a$ modulo $b$ and $\lambda_{i}$ is the stationary distribution of an $L$-state Markov chain and is defined as follows

$\lambda_{i}=\left[\sum_{j=0}^{L-1} \frac{F_{L-1}\left(\gamma_{T}\right)\left(1-F_{j}\left(\gamma_{T}\right)\right)}{F_{j}\left(\gamma_{T}\right)\left(1-F_{L-1}\left(\gamma_{T}\right)\right)}\right]^{-1} \frac{F_{L-1}\left(\gamma_{T}\right)\left(1-F_{i}\left(\gamma_{T}\right)\right)}{F_{i}\left(\gamma_{T}\right)\left(1-F_{L-1}\left(\gamma_{T}\right)\right)}$.

Similarly, by substituting (1) and (2) into (8), the PDF of the output SNR of an $L$-base station SEC combiner operating over i.n.i.d. Nakagami- $m$ channels can be obtained as given in (10).

\section{L-base Station SECps operating over Nakagami-m Fading Channels}

When the received SNR at the currently selected base station falls below the predetermined switching threshold a receiver utilizing an SECps scheme switches from the current base station to another base station and examines its SNR in exactly the same manner as an SEC scheme. However, when there is no acceptable base station available after examining all base stations, the SECps scheme selects the best performing base station, i.e., the base station with the highest SNR, instead of the last examined one. For an $L$-base station SECps system in which the SNR is i.n.i.d. at each of the $L$ base stations, the PDF of the output SNR can be expressed as [29] 


$$
f_{\mathrm{SSC}}(\gamma)=\left\{\begin{array}{cc}
\sum_{i=0}^{L-1}\left(\sum_{j=0}^{L-1} \frac{\Gamma\left(m_{j}\right)}{\Gamma\left(m_{j}\right)-\Gamma\left(m_{j}, m_{j} \gamma_{T} / \bar{\gamma}_{j}\right)}\right)^{-1} \frac{m_{i}^{m_{i}} \gamma^{m_{i}-1}}{\Gamma\left(m_{i}\right) \bar{\gamma}_{i}^{m_{i}}} \exp \left(-\frac{m_{i} \gamma}{\bar{\gamma}_{i}}\right), & \gamma<\gamma_{T} \\
\sum_{i=0}^{L-1}\left[\lambda_{i}+\left(\sum_{j=0}^{L-1} \frac{\Gamma\left(m_{j}\right)}{\Gamma\left(m_{j}\right)-\Gamma\left(m_{j}, m_{j} \gamma_{T} / \bar{\gamma}_{j}\right)}\right)^{-1}\right] \frac{m_{i}^{m_{i}} \gamma^{m_{i}-1}}{\Gamma\left(m_{i}\right) \bar{\gamma}_{i}^{m_{i}}} \exp \left(-\frac{m_{i} \gamma}{\bar{\gamma}_{i}}\right), & \gamma \geq \gamma_{T}
\end{array}\right.
$$

where $\lambda_{i}=\left(\sum_{j=0}^{L-1} \frac{\Gamma\left(m_{j}\right)}{\Gamma\left(m_{j}\right)-\Gamma\left(m_{j}, m_{j} \gamma_{T} / \overline{\gamma_{j}}\right)}\right)^{-1} \frac{\Gamma\left(m_{i}\right)}{\Gamma\left(m_{i}\right)-\Gamma\left(m_{i}, m_{i} \gamma_{T} / \overline{\gamma_{i}}\right)}, i=0,1, \ldots, L-1$.

$$
f_{\mathrm{SEC}}(\gamma)= \begin{cases}\sum_{i=0}^{L-1} \lambda_{i}\left[\prod_{k=0, k \neq i}^{L-1}\left(1-\frac{\Gamma\left(m_{k}, m_{k} \gamma_{T} / \overline{\gamma_{k}}\right)}{\Gamma\left(m_{k}\right)}\right)\right] \frac{m_{i}^{m_{i}} \gamma^{m_{i}-1}}{\Gamma\left(m_{i}\right) \bar{\gamma}_{i}^{m_{i}}} \exp \left(-\frac{m_{i} \gamma}{\bar{\gamma}_{i}}\right), & \gamma<\gamma_{T} \\ \sum_{i=0}^{L-1}\left[\sum_{j=0}^{L-1} \lambda_{(i-j)_{L}} \prod_{k=0}^{j-1}\left(1-\frac{\Gamma\left(m_{n}, m_{n} \gamma_{T} / \bar{\gamma}_{n}\right)}{\Gamma\left(m_{n}\right)}\right)\right] \frac{m_{i}^{m_{i}} \gamma^{m_{i}-1}}{\Gamma\left(m_{i}\right) \bar{\gamma}_{i}^{m_{i}}} \exp \left(-\frac{m_{i} \gamma}{\bar{\gamma}_{i}}\right), & \gamma \geq \gamma_{T}\end{cases}
$$

where $n=(i-j+k)_{L}$ and

$$
\lambda_{i}=\left[\sum_{j=0}^{L-1} \frac{\left(1-\frac{\Gamma\left(m_{L-1}, \frac{m_{L-1} \gamma_{T}}{\bar{\gamma}_{L-1}}\right)}{\Gamma\left(m_{L-1}\right)}\right)\left(\frac{\Gamma\left(m_{j}, \frac{m_{j} \gamma_{T}}{\bar{\gamma}_{j}}\right)}{\Gamma\left(m_{j}\right)}\right)}{\left(1-\frac{\Gamma\left(m_{j}, \frac{m_{j} \gamma_{T}}{\gamma_{j}}\right)}{\Gamma\left(m_{j}\right)}\right)\left(\frac{\Gamma\left(m_{L-1}, \frac{m_{L-1} \gamma_{T}}{\gamma_{L-1}}\right)}{\Gamma\left(m_{L-1}\right)}\right)}\right]^{-1} \frac{\left(1-\frac{\Gamma\left(m_{L-1}, \frac{m_{L-1} \gamma_{T}}{\bar{\gamma}_{L-1}}\right)}{\Gamma\left(m_{L-1}\right)}\right)\left(\frac{\Gamma\left(m_{i}, \frac{m_{i} \gamma_{T}}{\gamma_{i}}\right)}{\Gamma\left(m_{i}\right)}\right)}{\left(1-\frac{\Gamma\left(m_{i}, \frac{m_{i} \gamma_{T}}{\gamma_{i}}\right)}{\Gamma\left(m_{i}\right)}\right)\left(\frac{\Gamma\left(m_{L-1}, \frac{m_{L-1} \gamma_{T}}{\bar{\gamma}_{L-1}}\right)}{\Gamma\left(m_{L-1}\right)}\right)}
$$

$$
F_{\text {SECps }}(\gamma)=\left\{\begin{array}{cc}
\prod_{i=1}^{L} F_{i}(\gamma), & \gamma<\gamma_{T} \\
\prod_{k=1}^{L} F_{k}\left(\gamma_{T}\right) & \\
+\sum_{i=1}^{L}\left[\prod_{j=1}^{i-1} F_{j}\left(\gamma_{T}\right)\right]\left[F_{i}(\gamma)-F_{i}\left(\gamma_{T}\right)\right], & \gamma \geq \gamma_{T}
\end{array}\right.
$$

The PDF of the output SNR can then be obtained by differentiating (12) with respect to $\gamma$

$$
f_{\mathrm{SECps}}(\gamma)=\left\{\begin{array}{l}
\sum_{i=1}^{L}\left[\prod_{j=1}^{i-1} F_{j}\left(\gamma_{T}\right)\right] f_{i}(\gamma), \quad \gamma \geq \gamma_{T} \\
\sum_{i=1}^{L}\left[\prod_{j=1, j \neq i}^{L} F_{j}(\gamma)\right] f_{i}(\gamma), \quad \gamma<\gamma_{T}
\end{array}\right.
$$

Again, the PDF of the output SNR at an $L$-base station SECps combiner operating over i.n.i.d. Nakagami- $m$ channels can be obtained by substituting (1) and (2) into (13) as given in (14).

\section{ExPerimental Setup And MEAsurement PROCEDURE}

The wearable device used in this study was a purposely developed wireless sensor node with dimensions of $45 \mathrm{~mm} \mathrm{x}$ $60 \mathrm{~mm} \times 1.6 \mathrm{~mm}$ as shown in Fig. 1(a). The unit consisted of a CC1110F32 radio frequency (RF) transceiver, manufactured by Texas Instruments (TI) which was configured to operate at $868 \mathrm{MHz}$ using a printed meander-line PCB monopole antenna. During the experiments, the unit was configured to transmit a 9 byte data packet at a data rate of $500 \mathrm{Kbps}$ using minimum-shift keying. All data transmissions occurred at a regular interval of $20 \mathrm{~ms}$ with an output power level of $0 \mathrm{dBm}$.

The test subject (an adult male of height $1.70 \mathrm{~m}$ and mass $75 \mathrm{~kg}$ ) wore a sports T-shirt (86\% polyester / $14 \%$ elastane) with a special holding pocket purposely sown on to the garment at the central chest region as shown in Fig. 1(a). The sensor node was carefully positioned in the pocket such that the printed antenna was in a plane parallel to the surface of the test subject's body. It is worth highlighting that the battery which was used to power the sensor node ensured that there was at least a $6 \mathrm{~mm}$ separation between the body and the RF circuitry. The virtual base station array consisted of 10 identical, equidistant base stations which were positioned in a rectangular configuration with a length of approximately $20 \mathrm{~m}$ and a width of $6.6 \mathrm{~m}$ as shown in Fig. 2. The purposely 


$$
f_{\mathrm{SECps}}(\gamma)=\left\{\begin{array}{cl}
\sum_{i=1}^{L}\left[\prod_{j=1}^{i-1}\left(\frac{\Gamma\left(m_{j}, m_{j} \gamma_{T} / \bar{\gamma}_{j}\right)}{\Gamma\left(m_{j}\right)}\right)\right] \frac{m_{i}^{m_{i}} \gamma^{m_{i}-1}}{\Gamma\left(m_{i}\right) \bar{\gamma}_{i}^{m_{i}}} \exp \left(-\frac{m_{i} \gamma}{\bar{\gamma}_{i}}\right), \quad \gamma \geq \gamma_{T} \\
\sum_{i=1}^{L}\left[\prod_{j=1, j \neq i}^{L}\left(\frac{\Gamma\left(m_{j}, m_{j} \gamma / \bar{\gamma}_{j}\right)}{\Gamma\left(m_{j}\right)}\right)\right] \frac{m_{i}^{m_{i}} \gamma^{m_{i}-1}}{\Gamma\left(m_{i}\right) \bar{\gamma}_{i}^{m_{i}}} \exp \left(-\frac{m_{i} \gamma}{\bar{\gamma}_{i}}\right), & \gamma<\gamma_{T}
\end{array}\right.
$$

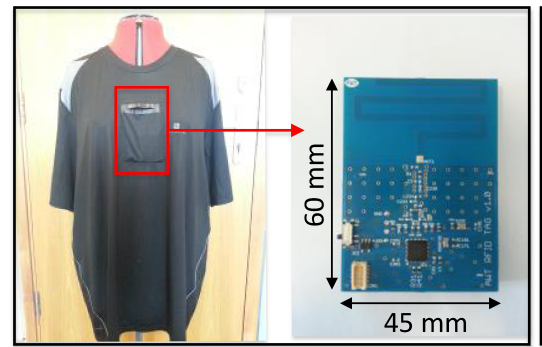

(a)

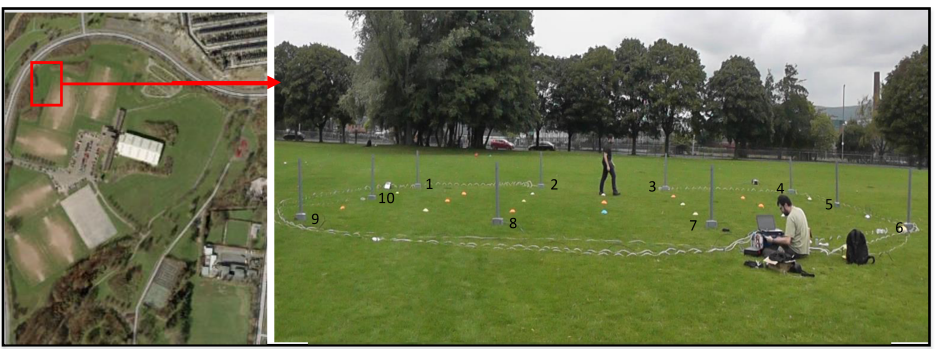

(b)

Fig. 1. (a) Purposely developed garment with mounting pocket at front central chest and the bodyworn wireless node used in the measurements; (b) satellite view of the measurement environment showing the positions of base stations.
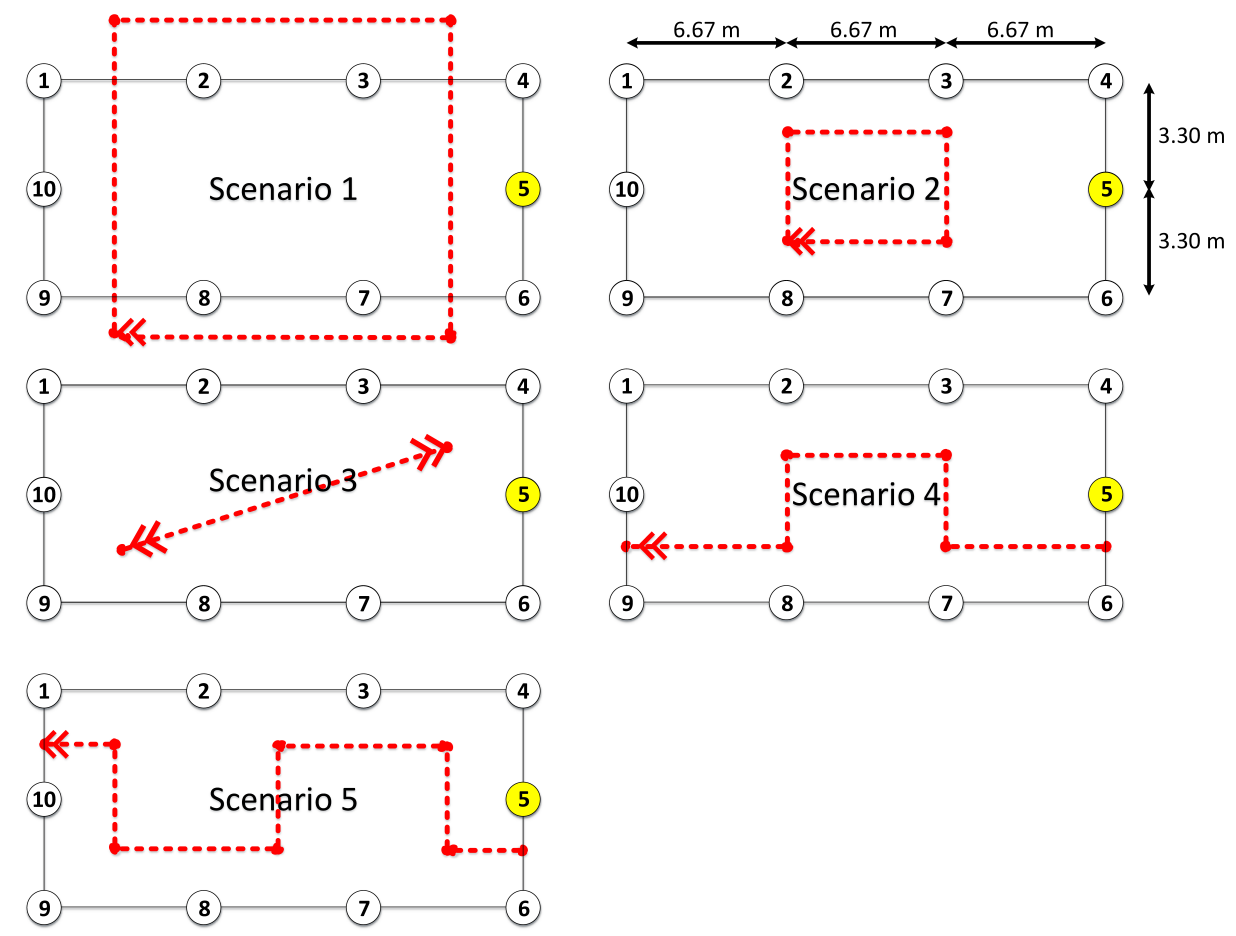

Fig. 2. Five individual user movement scenarios: a rectangular shape walk path (scenarios 1 and 2); a diagonal-line walk (scenario 3); a meandering walk path (scenarios 4 and 5). It should be noted that dotted lines and base station 5 represent the test subject's walk path and the target base station, respectively.

developed base station units also consisted of a CC1110F32 $\mathrm{RF}$ transceiver configured to record the received signal strength of each received packet. The antenna used by the base station units was a $+3.0 \mathrm{dBi}$ omnidirectional monopole antenna with a $10 \mathrm{~dB}$ bandwidth of $61 \mathrm{MHz}$ (833-894 MHz), positioned at a height of $1 \mathrm{~m}$ from ground level using a non-conductive support.
As shown in Fig. 1(b), all of the experiments conducted in this study were carried out in an outdoor playing field at the Ormeau Park within the city of Belfast in the United Kingdom. They were designed to emulate the channel characteristics which may be present in outdoor wearable applications such as sports and health monitoring, people tracking and positioning etc. Five individual measurement scenarios, all based around 
a walking test subject, were considered as shown in Fig. 2. These scenarios can be broadly categorized into the walk path which the movement followed. They included a rectangular shaped walk path (scenarios 1 and 2), a diagonal-line walk path (scenario 3) and a meandering walk path (scenarios 4 and 5). In practice, it is the SNR which is of most interest in the analysis of the performance of wireless systems. For the purpose of transforming the received signal power into the received SNR, prior to the acquisition of the channel data, a number of noise floor measurements were performed. To improve the robustness of the noise parameter estimates, these measurements were repeated 10 times. The corresponding mean recorded noise threshold was $-100.9 \mathrm{dBm}$ while the corresponding lowest received signal power observed in the environment was $-94.0 \mathrm{dBm}$.

\section{Base Station Correlation, Diversity Gain, Number OF PATH EXAMINATIONS AND NUMBER OF PATH SWITCHES}

Prior to the data analysis, it was required to transform the received signal power into the received SNR. To this end, the noise parameters $\mu$ (mean) and $\sigma$ (standard deviation) were first estimated from noise floor measurements using maximum likelihood estimation (MLE) [30] under the assumption that noise envelope was characterized by a Gaussian distribution [31]. The estimated noise parameters obtained over the 10 repeated trials were then averaged and found to be $0.0021 \mathrm{mV}$ (standard deviation $0.017 \mathrm{uV}$ ) and $0.0006 \mathrm{mV}$ (standard deviation $0.009 \mathrm{uV}$ ) for the $\mu$ and $\sigma$ parameters, respectively. The total duration of the 10 repeated noise floor measurements was approximately 10 minutes. From the noise parameter estimates, random variables representing the noise process were then generated. Following from this, the received signal power was then transformed into the received SNR using the definition of SNR, SNR $=\mathrm{P}_{\text {Signal }} / \mathrm{P}_{\text {Noise }}$ where $\mathrm{P}_{\text {Signal }}$ and $\mathrm{P}_{\text {Noise }}$ denote the signal power and noise power, respectively. As an example, Fig. 3 shows the received signal power at base station 1 for scenario 5 along with its transformed SNR. The average received SNR at each base station for all considered scenarios ranged between $22.6 \mathrm{~dB}$ and $34.0 \mathrm{~dB}$. Based on these averaged SNR values, three different switching thresholds were determined, which were low $(10 \mathrm{~dB})$, medium $(30 \mathrm{~dB})$ and high $(50 \mathrm{~dB})$ thresholds.

\section{A. Base Station Correlation}

Since any significant correlation between the signal received at each of the base stations has the potential to degrade the diversity gain, it is important to determine the level of correlation which may exist. For a macro-diversity scheme to be effective, the received signal at each base station should be statistically independent. In the context of diversity reception, two signals are said to be suitably de-correlated if their crosscorrelation coefficient is less than 0.7 [1]. Fig. 4 shows the CDFs of the cross-correlation coefficients calculated for all of the considered scenarios. It is worth noting that the crosscorrelation coefficient computations were performed using the approach proposed in [32]. It is clear from the Fig. 4 that the

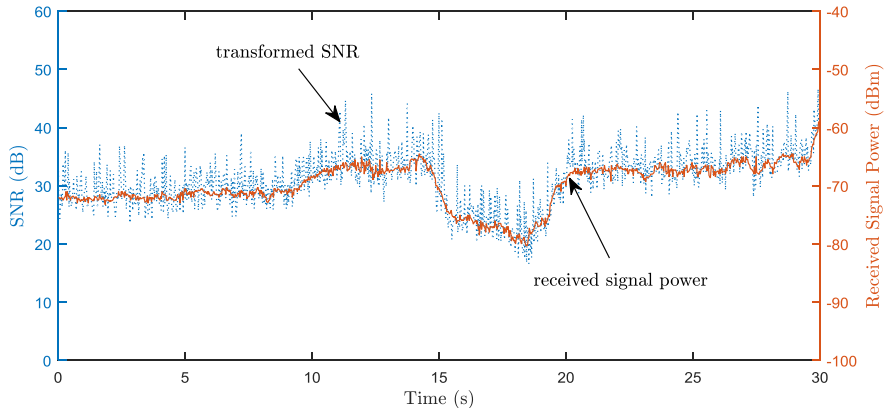

Fig. 3. Received signal power (continuous lines) at base station 1 for scenario 5 alongside the transformed SNR (dashed lines).

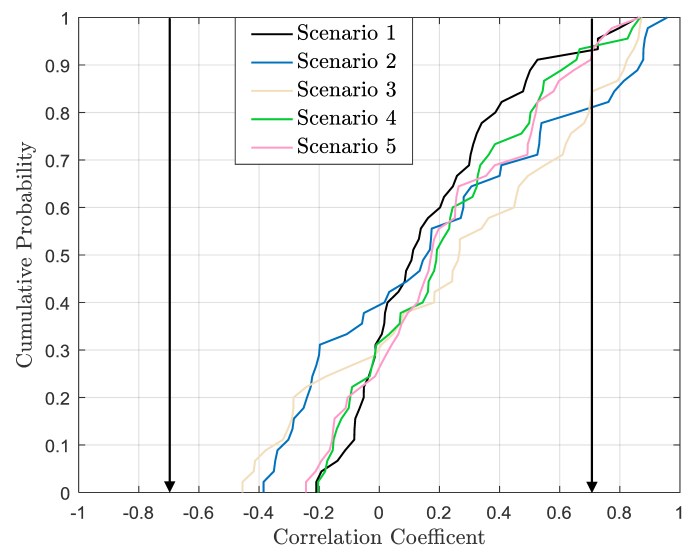

Fig. 4. CDFs of the calculated cross-correlation coefficients for all of the considered scenarios.

majority of the estimated cross-correlation coefficients were between -0.7 and 0.7 . This result suggests that a switched combining based macro-diversity system equipped with multiple base stations can provide a worthwhile improvement in the received signal reliability.

\section{B. Diversity Gain}

To evaluate the potential improvement in the received signal reliability that could be obtained using a switched combining based macro-diversity system with the SSC, SEC and SECps schemes, we utilize the concept of macro-diversity gain. This is defined as the signal reliability improvement between the SNR of the diversity combiner and that of the target base station for a given probability or signal reliability. It should be noted that for this study, base station 5 was considered as the target base station and all macro-diversity gain calculations were made at a signal reliability of $90 \%$. For brevity, herein we refer to macro-diversity gain as simply diversity gain. In this study, we have considered dual- and four-base station configurations with two different potential groupings of base stations. These were: group 1 a dual-base station configuration consisting of base stations 1 and 6; group 2 another dualbase station configuration consisting of base stations 5 and 10; group 3 a four-base station configuration consisting of base stations 1, 4, 6 and 9; group 4 another four-base station configuration consisting of base stations $3,5,8$ and 10 . 
TABLE I

Diversity GAINS FOR THE SSC, SEC, SECPS AND PSC SCHEMES WITH THREe DiFFERENT Switching ThresholdS FOR ALl MEASUREMENT SCENARIOS AND GROUPINGS.

\begin{tabular}{|c|c|c|c|c|c|c|c|c|c|c|c|c|c|c|c|}
\hline & \multirow{3}{*}{$\begin{array}{c}\text { Switching } \\
\text { Threshold } \\
\text { (dB) }\end{array}$} & \multicolumn{14}{|c|}{ Macro-Diversity Gain (dB) } \\
\hline & & \multicolumn{4}{|c|}{ Group $1(1,6)$} & \multicolumn{4}{|c|}{ Group $2(5,10)$} & \multicolumn{3}{|c|}{ Group $3(1,4,6,9)$} & \multicolumn{3}{|c|}{ Group $4(3,5,8,10)$} \\
\hline & & $\mathrm{SSC}$ & SEC & SECps & PSC & $\mathrm{SSC}$ & SEC & SECps & PSC & SEC & SECps & PSC & SEC & SECps & PSC \\
\hline \multirow[t]{3}{*}{ S1 } & 30 & 2.8 & 2.8 & 10.6 & 10.6 & -0.9 & -0.9 & 6.2 & 6.2 & 13.7 & 13.7 & 13.7 & 12.3 & 13.2 & 13.2 \\
\hline & 50 & -0.4 & -0.4 & 10.6 & & -2.2 & -2.2 & 6.2 & & 0.2 & 13.7 & & -1.0 & 13.2 & \\
\hline & 10 & -2.5 & -2.5 & -2.5 & & 0.0 & 0.0 & 0.0 & & -2.5 & -2.5 & & -3.6 & -3.6 & \\
\hline \multirow{2}{*}{ S2 } & 50 & -8.4 & -8.4 & 6.4 & & -8.5 & -8.5 & 5.1 & & -8.2 & 6.9 & & -6.5 & 7.8 & \\
\hline & 10 & 6.2 & 6.2 & 6.2 & & 1.9 & 1.9 & 1.9 & & 1.1 & 1.1 & & 1.8 & 1.8 & \\
\hline \multirow[t]{3}{*}{$\mathbf{S 3}$} & 30 & 9.8 & 9.8 & 12.8 & 12.8 & 3.2 & 3.2 & 11.4 & 11.4 & 14.6 & 14.7 & 14.9 & 14.9 & 14.9 & 15.0 \\
\hline & 50 & 3.5 & 3.5 & 12.8 & & 0.4 & 0.4 & 11.4 & & -2.0 & 14.9 & & 0.2 & 15.0 & \\
\hline & 10 & 8.0 & 8.0 & 8.0 & & 9.6 & 9.6 & 9.6 & & 12.2 & 12.2 & & 9.3 & 9.3 & \\
\hline S5 & 50 & 5.5 & 5.5 & 16.6 & & 3.7 & 3.7 & 15.0 & & 4.0 & 18.4 & & 5.1 & 18.3 & \\
\hline
\end{tabular}

Table I shows the diversity gain statistics for dual-base station SSC, SEC and SECps schemes with groups 1 and 2, four-base station SEC and SECps schemes with groups 3 and 4. For comparison, the diversity gains for dual- and fourbase station PSC are also shown in Table I. For a medium switching threshold, the benefit of having more than two available base stations was demonstrated by comparing the diversity gains between dual- and four-base station configurations. For example, four-base station SEC with group 3 for scenario 1 provided a diversity gain of $13.7 \mathrm{~dB}$ whereas dualbase station SEC with group 1 achieved a diversity gain of $2.8 \mathrm{~dB}$. Moreover, for all of the groupings and scenarios, dualbase station SEC provided the same performance as dual-base station SSC. Table I shows that the estimated diversity gain not only varied according to the different scenarios but also with different groupings. This observation can be attributed to each base station having experienced different shadowing intensities depending on the position and orientation of the test subject's body during each movement scenario. When comparing the diversity gains obtained across all scenarios, the values obtained for scenario 4 were the highest. One possible explanation for this is that the average SNR at the target base station (base station 5) for scenario 4 was smaller than those for the other scenarios. This occurred due to the direct signal path between the bodyworn node and the target base station existing only for a relatively short length of time compared to those for other scenarios due to the test subject's movement.

To visually investigate the improvement in the received signal reliability using the switched diversity schemes, Fig. 5 shows the received SNR time series at base stations 1, 4, 6 and 9 (group 3) along with the output SNR of four-base station SEC and SECps with a medium switching threshold $(30 \mathrm{~dB})$ for scenario 2 . When the currently selected base station suffered from deep fading due to the shadowing caused
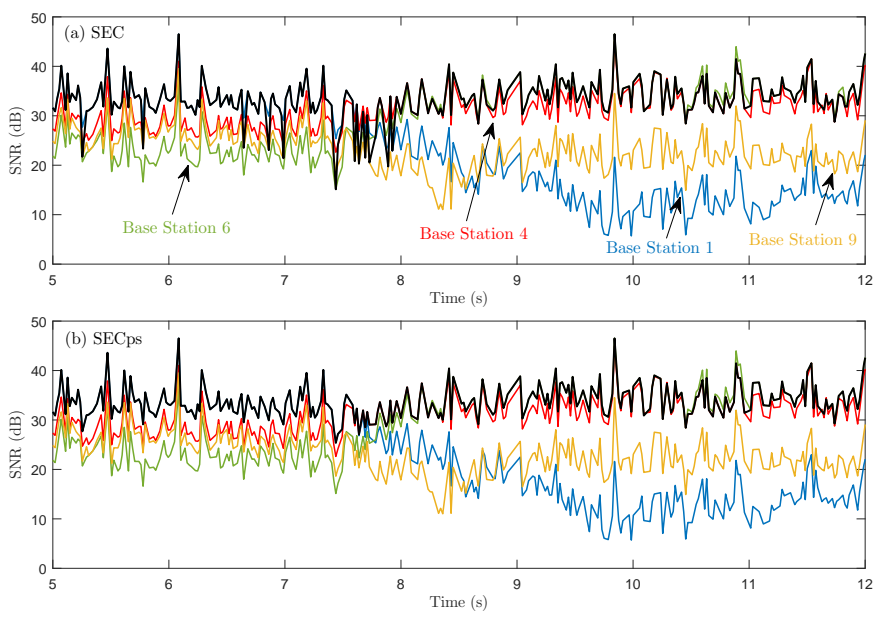

Fig. 5. Received SNR at base stations 1 (blue), 4 (red), 6 (green) and 9 (yellow) along with the output SNR (black) of four-base station switched diversity schemes for scenario 2: (a) SEC and (b) SECps.

by the test subject's body, the switched combining based macro-diversity schemes selected and switched to another base station. Consequently, for both the SEC and SECps schemes, the majority of SNR values below the $30 \mathrm{~dB}$ level (the predetermined switching threshold) were eliminated. This suggests that a macro-diversity system using either an SEC or SECps scheme made up of base stations 1, 4, 6 and 9 can mitigate shadowing effect of the person's body and achieve a significant improvement in signal reliability.

For a dual-base station configuration operating with a medium switching threshold, group 1 always provided a greater diversity gain than group 2 with the exception of scenario 4. This suggests that the location of the base stations in group 1 was more appropriate compared to group 2 


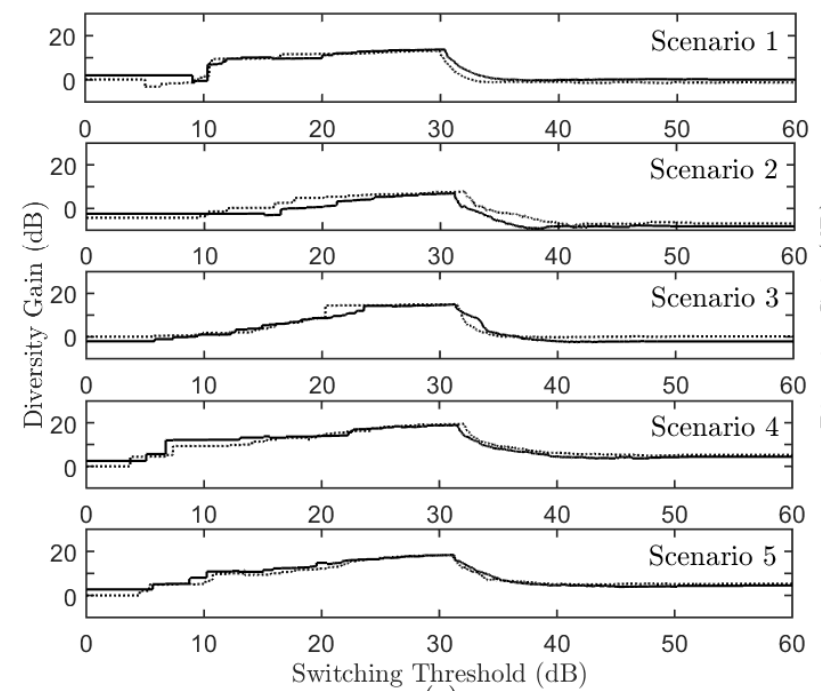

(a)

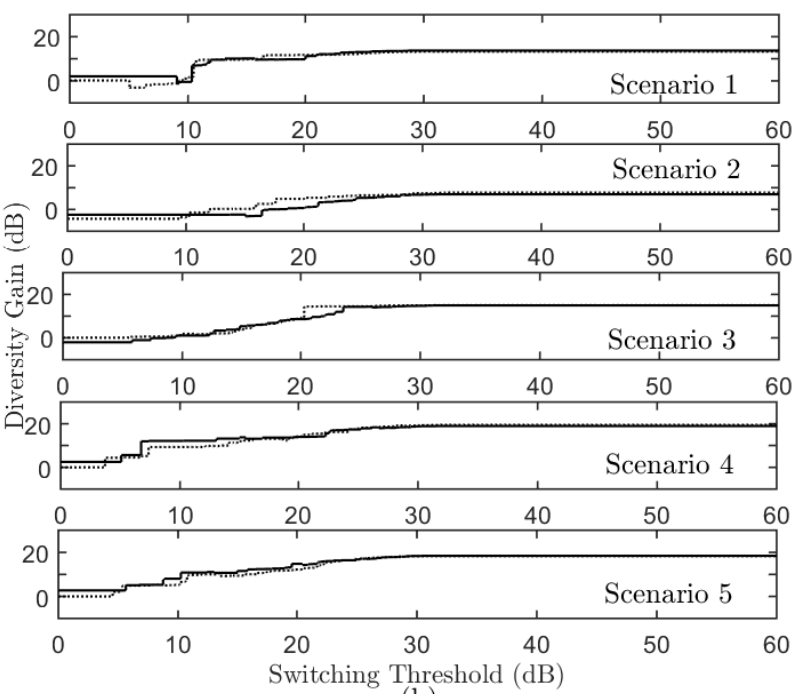

(b)

Fig. 6. Effect of different switching thresholds on achievable diversity gain of four-base station (a) SEC and (b) SECps with group 3 (continuous lines) and group 4 (dashed lines) for all of movement scenarios.

in general. However, for a four-base station configuration, groups 3 and 4 provided similar diversity gains for all of the movement scenarios. As well as this, for the majority of the movements considered in this study, adding the two additional base stations also offered no significant difference in the diversity gains provided by the SEC and SECps schemes when a medium switching threshold was selected. For example, as shown in Table I, the diversity gains for dual-base station SEC and SECps with group 1 for scenario 1 were $2.8 \mathrm{~dB}$ and $10.6 \mathrm{~dB}$, respectively. On the other hand, for four-base station SEC and SECps with group 3, both had the same diversity gain which was $13.7 \mathrm{~dB}$. These suggest that a switched diversity system with more than two base stations is less affected by the location of the base stations and thus provides a more stable performance in terms of signal reliability.

Furthermore, it was found that the estimated diversity gains for both the dual-base station and four-base station configurations strongly depended on the switching threshold. For example, the diversity gains for four-base station SEC with group 3 for scenario 2 were $-2.5,6.8$ and $-8.2 \mathrm{~dB}$ at the low, medium and high switching thresholds, respectively. Interestingly, negative diversity gains were also observed at the low and high switching thresholds, indicating that there was no benefit to using a switched diversity scheme with these switching thresholds. To examine the effect of different switching thresholds on the achievable diversity gain, Fig. 6 shows the diversity gains for four-base station SEC and SECps with different switching threshold values ranging between $0 \mathrm{~dB}$ and $60 \mathrm{~dB}$ for all of scenarios. It is obvious that the estimated diversity gains varied according to the switching threshold, but there always existed an optimum switching threshold which maximizes the diversity gain. With the optimum switching threshold, both four-base station SEC and SECps systems provided almost the same diversity gain as an equivalent PSC system. While the diversity gain for four-base station
SEC decreases beyond the optimum switching threshold, the diversity gain for four-base station SECps became saturated at the maximum achievable diversity gain level. This was most likely due to the different combining methods implemented by the SEC and SECps schemes. The SEC scheme selects the last examined base station when no acceptable base station is found after examining all available options. On the other hand, the SECps scheme selects the best path which has the highest SNR among all paths instead of the last examined base station. The SECps scheme therefore acts as a PSC scheme when the switching threshold is high and no acceptable path is found after examining all diversity paths.

\section{Number of Path Examinations and Switches}

To evaluate the trade off between performance and complexity (which is related to energy consumption), we evaluated the number of path examinations and the number of path switches between base stations for the SEC and SECps schemes. As shown in Fig. 7, the number of path examinations and switches for the SEC an SECps schemes were almost the same when the switching threshold was low while the SECps scheme had a greater number of path examinations and switches compared to the SEC scheme at the middle and high switching threshold levels. This was most likely due to that when there is no acceptable base station available the SEC scheme usually selects the last examined base station while the SECps scheme selects the best performing base station. Consequently, for an $L$-base station arrangement, the SEC scheme has at most $L-1$ path switches whereas the SECps scheme has at most $L$ path switches, i.e., the number of path switches for the SECps scheme usually is greater than that for the SEC scheme. They are the same only if the last examined base station has the highest SNR.

Likewise, the number of path examinations for the SEC and SECps schemes were almost the same at the low switching 

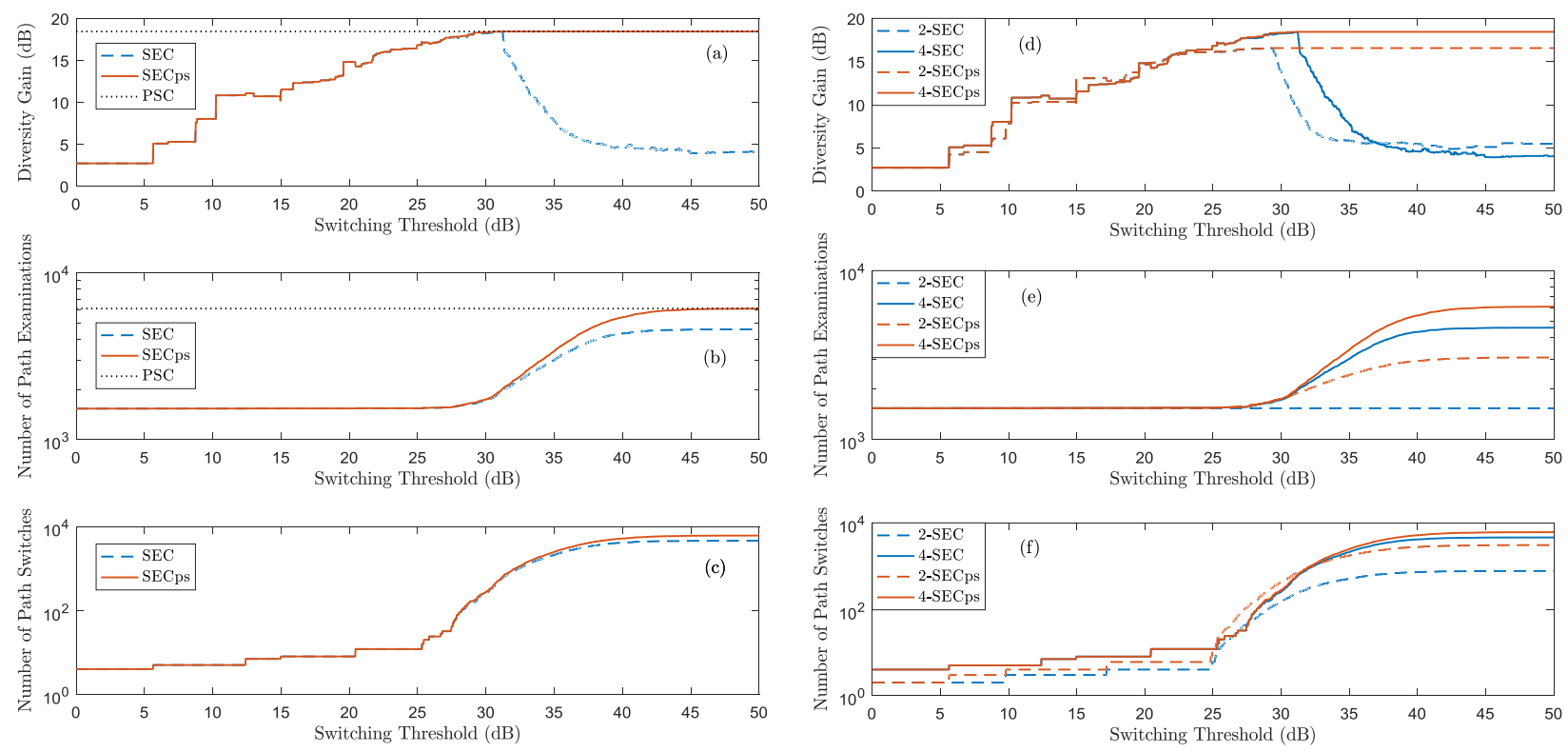

Fig. 7. Diversity gain, the number of path examinations and the number of path switches for scenario 5 using the group 3 configuration with four-base station SEC and SECps schemes for different switching thresholds. For comparison, (a)-(b) four-base station PSC using the group 3 configuration and (d)-(f) dual-base station SEC and SECps using the group 1 configuration are also presented.

threshold levels whereas the number of path examinations for the SECps was greater than that for the SEC at the medium and high switching threshold levels. More specifically, for an $L$-base station arrangement, when the receiver switches from the $(L-1)^{\text {th }}$ base station to the $L^{\text {th }}$ base station (i.e., last base station), the SECps scheme needs to monitor the $L^{\text {th }}$ base station whereas the SEC scheme does not need to do this because it switches to the $L^{\text {th }}$ base station anyway irrespective of whether the received SNR at $L^{\text {th }}$ base station is above the switching threshold or not. Based on the diversity gain, the number of path examinations and the number of path switches, it was observed that the SECps scheme provided a greater diversity gain, but the additional number of path examinations and switches will lead to more energy consumption compared to the SEC scheme.

When compared with a PSC scheme, as shown in Figs. 7(a) and (b), the SECps scheme had a smaller number of path examinations at medium switching threshold levels, but it had a similar diversity gain. This can be explained by the fact that the PSC scheme monitors the SNR of all of the base stations and then selects the base station with the highest SNR. Therefore, at medium switching threshold levels, the SECps scheme prevents the unnecessary monitoring while providing a similar performance compared to the PSC scheme, suggesting that the SECps scheme is less complex and thus more energy efficient compared to the PSC scheme. However, as expected, it was observed that the number of path examinations for the SECps and PSC became similar at high switching threshold levels. Figs. 7(d)-(f) show a comparison of the diversity gain, the number of path examinations and the number of path switches between base stations for dual- and four-base station SEC and SECps schemes. While it is obvious that four-base station SEC and SECps provided a greater diversity gain (higher performance) than dual-base station SEC and SECps, they also had a significantly greater number of path examinations and switches (higher complexity and higher energy consumption). Therefore the choice as to which configuration and scheme to implement for wearable systems will come down to a choice between performance and complexity. For some, but not all cases (see Fig. 7 and Table I), a dual-base station configuration with the appropriate switching threshold/technology may offer a good compromise between complexity (energy consumption) and information recovering capability.

\section{Fading Statistics at the OUtPut of the SWITCHED COMBINERS}

To characterize the fading behavior at the output of the virtual switched combiners, the diversity specific analytical equations presented in (6), (10) and (14) for $L$-base station SSC, SEC and SECps schemes operating in i.n.i.d. Nakagami$m$ fading channels were fitted to the empirical data. The Nakagami $m$ and $\bar{\gamma}$ parameters were estimated using a nonlinear least squares routine written in MATLAB to fit (6), (10) and (14) to the measurement data. Again, we have considered dual- and four-base station configurations with the same groupings presented in Section IV. It should be noted that the minimum data set size used for the parameter estimation was 761 samples.

As an example of the results of the model fitting process, Fig. 8 shows the PDFs of four-base station SEC and SECps (group 3) with three different switching thresholds for scenario 5. The resistor-average distance (RAD) was evaluated to provide a quantitative measure of the goodness-of-fit of the theoretical PDFs with the measured data. Unlike the KullbackLeibler divergence (KLD), the RAD satisfies the triangle inequality and thus is a true distance metric. This can be defined as follows [33] 

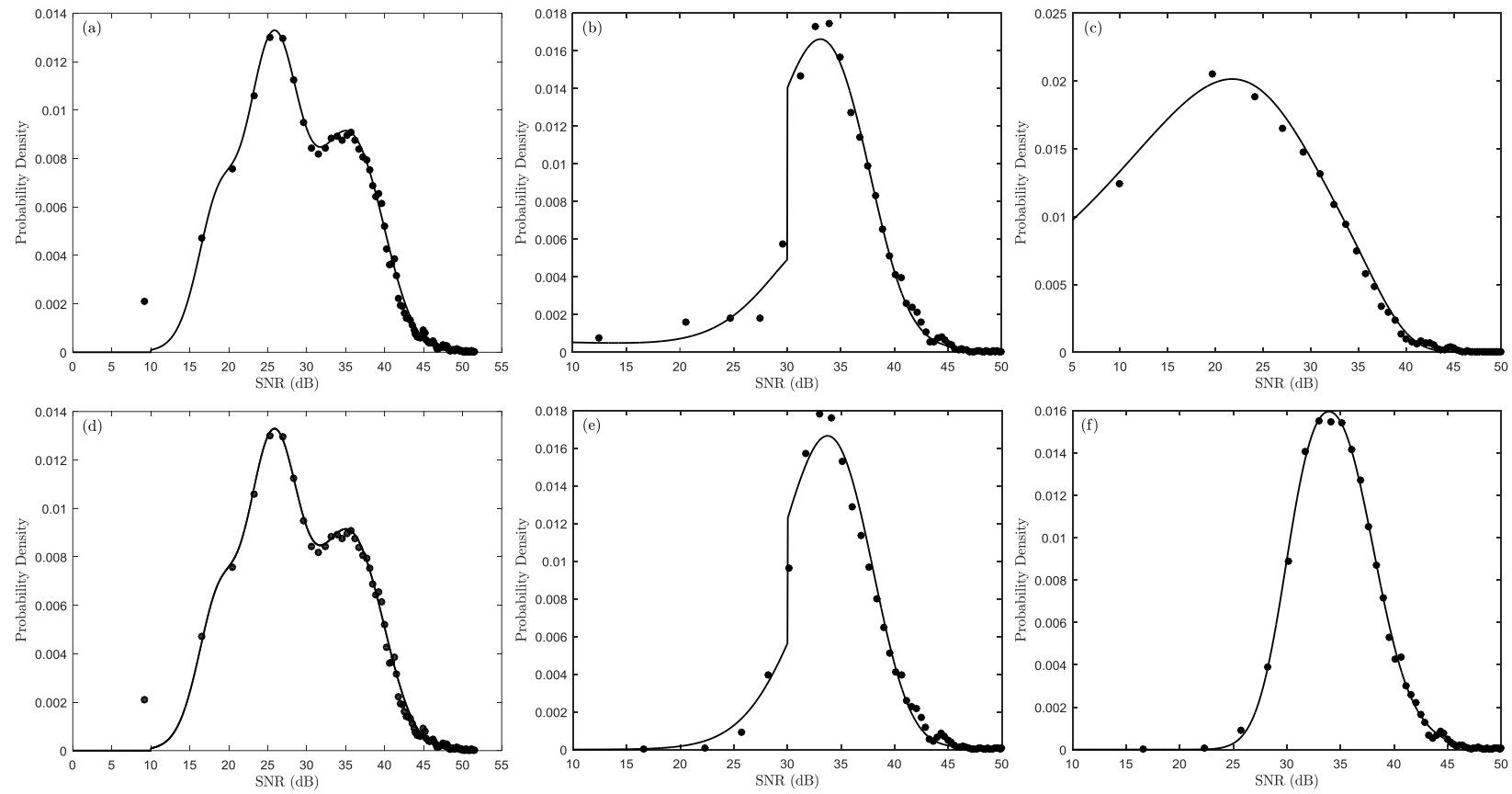

Fig. 8. Empirical (black circle symbols) and theoretical (continuous lines) PDFs for scenario 5 with group 3 for four-base station SEC with (a) low, (b) medium and (c) high switching thresholds and four-base station SECps with (d) low, (e) medium and (f) high switching thresholds.

$$
\operatorname{RAD}=\left(\frac{1}{\operatorname{KLD}(p, q)}+\frac{1}{\operatorname{KLD}(q, p)}\right)^{-1}
$$

where $\operatorname{KLD}(\cdot, \cdot)$ denotes the $\operatorname{KLD}$ given by $\operatorname{KLD}(p, q)=$ $\int_{-\infty}^{\infty} p(x) \log _{2}\left(\frac{p(x)}{q(x)}\right) d x$ with $p(x)$ and $q(x)$ denoting the true PDF of the data and the test PDF, i.e., the approximated PDF of $p(x)$, respectively [34]. As the RAD tends towards zero, the test PDF (theoretical PDF) is in very good agreement with the true PDF (empirical PDF). The RAD analysis results are presented in Table II. The minimum and maximum RAD values were 0.0039 and 0.0887 respectively, suggesting that the theoretical models for SEC and SECps generally provided an adequate fit to the measured data. To assist with the interpretation of the goodness-of-fit, the equivalent RAD was used to calculate the standard deviation $\sigma$ of a zero-mean, $\sigma^{2}$ variance Gaussian PDF that is used to approximate a zeromean, unit variance Gaussian PDF $^{1}$. The minimum and maximum calculated $\sigma$ values corresponding to the RAD above were 1.09 and 1.53 respectively. As the Gaussian distribution is widely known and used, this provides a reference level upon which our fits may be interpreted.

Furthermore, to allow the reader to reproduce the theoretical plots reported, Table III provides the parameter estimates for four-base station SEC and SECps (group 3) with three different switching thresholds for all considered movement scenarios. As we can see from Table III, the parameter estimates ob-

${ }^{1}$ For the case when $p$ is a Gaussian PDF with zero-mean and unit variance and $q$ is a Gaussian PDF with zero-mean and variance $\sigma^{2}$, we can have $\operatorname{KLD}(p, q)=0.5\left(\sigma^{-2}-1\right)+\ln (\sigma)$ and $\operatorname{KLD}(q, p)=0.5\left(\sigma^{2}-1\right)-$ $\ln (\sigma)$. By substituting these equations into (15), we can obtain as RAD $=$ $\left(\frac{1}{0.5\left(\sigma^{-2}-1\right)+\ln (\sigma)}+\frac{1}{0.5\left(\sigma^{2}-1\right)-\ln (\sigma)}\right)^{-1}$. tained for the fading experienced at each of the considered base stations were different, suggesting that each base station experienced non-identical Nakagami- $m$ fading. Additionally, as expected, the parameter estimates also varied according to the user's movement. For example, when comparing scenarios 4 and 5 for four-base station SECps with a medium switching threshold $(30 \mathrm{~dB})$, the estimated $m$ parameters for scenario 4 were higher than those for scenario 5 . This suggests that the fading observed at the four base stations during scenario 4 were subject to less shadowing compared to scenario 5. This is supported by the fact that the average SNR for the output of four-base station SECps (group 3) for scenario 4 (36.4 dB) was greater than that for scenario $5(35.8 \mathrm{~dB})$.

As shown in Fig. 8, the shapes of the PDFs were different according to the switching threshold that was adopted. When a low switching threshold was chosen (i.e., $10 \mathrm{~dB}$ ) the PDFs of both the SEC and SECps were the same as shown in Fig. 8 (a) and (d). This was most likely due to the fact that path switching between base stations rarely occurred at the low switching threshold level. More specifically, as shown in Fig. 9 (a), there was only one instance of path switching between base stations (base station $6 \rightarrow 9$ ) with the virtual switched combiner selecting base station 9 for the remainder of the time. It should be noted that the received SNR at base stations 1 and 4 in group 3 is not shown in Fig. 9(a) for clarity. As evidence for this, Fig. 9(b) shows that the PDF of the output SNR of four-base station SEC and the PDF of the received SNR at base station 9. It is clear that these two PDFs were similar. Note that the PDF of the received SNR at base station 9 in Fig. 9(b) was generated using the received SNR beyond the vertical line in Fig. 9(a) which represents path switching from base station 6 to 9 .

On the contrary, path switching between base stations occurs 
TABLE II

Estimated Resistor-Average Distance for All of the Considered Cases.

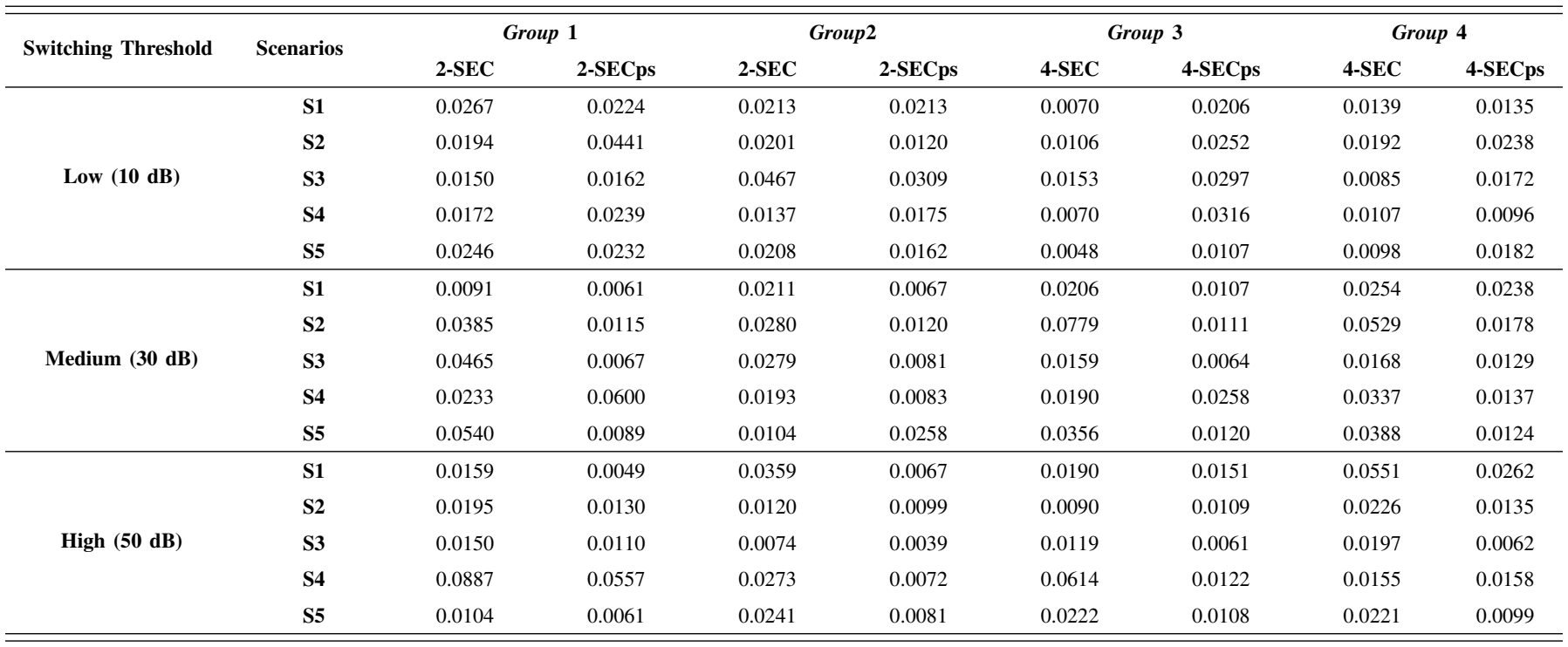
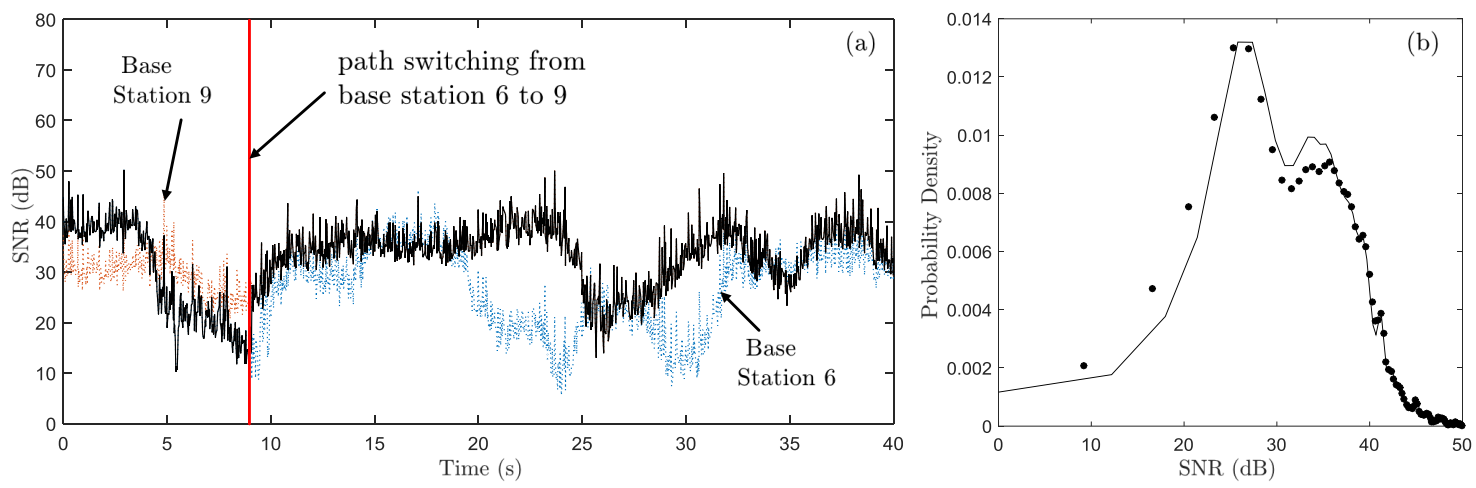

Fig. 9. (a) Received SNR at base stations 6 and 9 along with the output SNR of four-base station SEC (continuous lines); (b) PDFs of the output SNR (circle symbols) of four-base station SEC and the received SNR (continuous lines) at base station 9 beyond the vertical line which represents path switching from base station 6 to 9. It should be noted that the received SNR at base stations 1 and 4 in group 3 are not shown in Fig. 10(a) for clarity.

during almost every single time slot when the switching threshold is high $(5 \mathrm{~dB})$. This is particularly evident from Fig. 10, where the SEC scheme switched from the currently selected base station to another base station even if the presently selected base station had the highest SNR, causing a degradation in the overall performance. As a result of this, there was a significant increase in the spread of the output SNR of the SEC scheme as shown in Fig. 8(c). However, the SECps scheme does not follow this regime when the switching threshold is high as it favors the base station with the highest SNR when no acceptable signal base station is above the switching threshold (Fig. 10). As discussed in Section IV, in this scenario, the SECps scheme effectively operates as a PSC scheme. Therefore, unlike the SEC scheme, the majority of the SECps output SNR levels where in the reduced range from $25 \mathrm{~dB}$ to $45 \mathrm{~dB}$, which resulted in an empirical probability density with a significantly reduced spread as shown in Fig. 8(f).

For both the SEC and SECps schemes, when the switching threshold was set at a medium value $(30 \mathrm{~dB})$, the majority of combiner output SNR levels ranged between $25 \mathrm{~dB}$ and $45 \mathrm{~dB}$ as shown in Fig. 8(b) and (e). These were almost identical to those obtained for the SECps scheme configured with a high switching threshold, i.e., a PSC scheme, although occasionally the SEC scheme still had instances where the combiner output SNR was below $15 \mathrm{~dB}$. When compared in terms of the number of path examinations and achievable diversity gain, the SECps scheme operating with a medium switching threshold provided a more favorable alternative because it achieved sufficient performance with a lesser number of path examinations compared to the PSC scheme.

Further evidence of the benefits of having more than two base stations can also be provided by analyzing the fading statistics at the output of the switched diversity combiner. For example, Fig. 11 shows the PDFs of dual-base station SEC and SECps with group 2 and four-base station SEC and SECps with group 4 for scenario 2 when a medium switching threshold was adopted. As we can see, for both the SEC and SECps schemes, the four-base station configuration had a lower number of signal observations at low SNR levels and 
TABLE III

Parameter Estimates for Four-Base Station SEC AND SECps with Three Different Switching Thresholds For All of the COnsidered Movement Scenarios in Group 3 (BASE Stations 1, 4, 6, And 9).

\begin{tabular}{|c|c|c|c|c|c|c|c|c|c|c|c|c|c|c|c|c|}
\hline & \multicolumn{8}{|c|}{ 4-SEC } & \multicolumn{8}{|c|}{ 4-SECps } \\
\hline & $m_{1}$ & $\overline{\gamma_{1}}$ & $m_{2}$ & $\overline{\gamma_{2}}$ & $m_{3}$ & $\overline{\gamma_{3}}$ & $m_{4}$ & $\overline{\gamma_{4}}$ & $m_{1}$ & $\overline{\gamma_{1}}$ & $m_{2}$ & $\overline{\gamma_{2}}$ & $m_{3}$ & $\overline{\gamma_{3}}$ & $m_{4}$ & $\overline{\gamma_{4}}$ \\
\hline & \multicolumn{16}{|c|}{ Low Switching Threshold (10 dB) } \\
\hline S1 & 2.9 & 52.7 & 6.4 & 13.3 & 15.0 & 6.8 & 1.6 & 2.9 & 0.9 & 46.7 & 12.8 & 11.2 & 15.0 & 54.6 & 4.4 & 3.8 \\
\hline S2 & 9.0 & 14.6 & 4.1 & 59.4 & 15.0 & 7.1 & 15.0 & 9.0 & 1.5 & 53.5 & 15.0 & 13.2 & 12.0 & 13.2 & 14.1 & 7.9 \\
\hline $\mathbf{S 3}$ & 2.1 & 100 & 4.2 & 39.3 & 9.3 & 11.6 & 15.0 & 2.1 & 1.3 & 34.0 & 15.0 & 2.0 & 2.1 & 6.3 & 15.0 & 2.3 \\
\hline S4 & 8.0 & 14.3 & 3.8 & 64.2 & 15.0 & 7.1 & 15.0 & 7.5 & 1.8 & 68.1 & 15.0 & 18.8 & 9.3 & 16.9 & 14.1 & 88.1 \\
\hline \multirow[t]{2}{*}{ S5 } & 4.2 & 73.6 & 3.1 & 100 & 7.5 & 21.1 & 15.0 & 8.7 & 1.6 & 61.4 & 15.0 & 10.8 & 15.0 & 62.9 & 7.6 & 15.4 \\
\hline & \multicolumn{16}{|c|}{ Medium Switching Threshold (30 dB) } \\
\hline S1 & 3.9 & 58.3 & 0.5 & 0.1 & 10.0 & 1.5 & 0.8 & 62.9 & 4.2 & 61.2 & 0.5 & 48.9 & 15.0 & 7.1 & 15.0 & 7.1 \\
\hline $\mathbf{S 2}$ & 2.5 & 100 & 15.0 & 44.5 & 8.2 & 64.2 & 15.0 & 44.5 & 13.4 & 0.4 & 0.7 & 38.0 & 9.0 & 53.3 & 9.6 & 0.1 \\
\hline $\mathbf{S 3}$ & 15.0 & 1.8 & 11.7 & 1.1 & 0.6 & 80.6 & 6.1 & 58.1 & 0.5 & 30.5 & 7.6 & 59.7 & 15.0 & 19.6 & 15.0 & 19.6 \\
\hline $\mathbf{S 4}$ & 9.8 & 64.7 & 0.9 & 100 & 3.5 & 100 & 15.0 & 46.6 & 0.5 & 45.8 & 15.0 & 10.9 & 9.7 & 62.6 & 15.0 & 14.3 \\
\hline \multirow[t]{2}{*}{ S5 } & 0.8 & 62.8 & 4.7 & 58.9 & 15.0 & 1.0 & 9.1 & 4.8 & 5.1 & 60.9 & 0.5 & 100 & 2.9 & 0.4 & 8.9 & 0.2 \\
\hline & \multicolumn{16}{|c|}{ High Switching Threshold (50 dB) } \\
\hline S1 & 14.0 & 59.7 & 1.8 & 22.9 & 13.5 & 41.5 & 11.6 & 74.5 & 5.8 & 33.4 & 15.0 & 9.4 & 3.3 & 55.6 & 0.5 & 31.2 \\
\hline S2 & 1.7 & 25.1 & 8.1 & 32.9 & 12.9 & 83.1 & 5.0 & 51.3 & 7.6 & 26.9 & 5.1 & 51.0 & 15.0 & 34.1 & 0.5 & 30.4 \\
\hline S3 & 7.3 & 20.7 & 1.5 & 18.9 & 13.7 & 28.6 & 5.9 & 51.7 & 6.2 & 19.9 & 15.0 & 29.8 & 6.7 & 56.6 & 0.5 & 30.6 \\
\hline S4 & 8.1 & 49.1 & 13.7 & 35.4 & 5.5 & 58.5 & 1.7 & 26.2 & 6.8 & 58.7 & 14.9 & 32.5 & 0.5 & 21.0 & 0.5 & 41.7 \\
\hline S5 & 4.6 & 25.0 & 7.3 & 22.9 & 6.1 & 64.2 & 1.7 & 29.4 & 5.2 & 52.7 & 12.0 & 28.0 & 15.0 & 11.8 & 1.4 & 50.6 \\
\hline
\end{tabular}

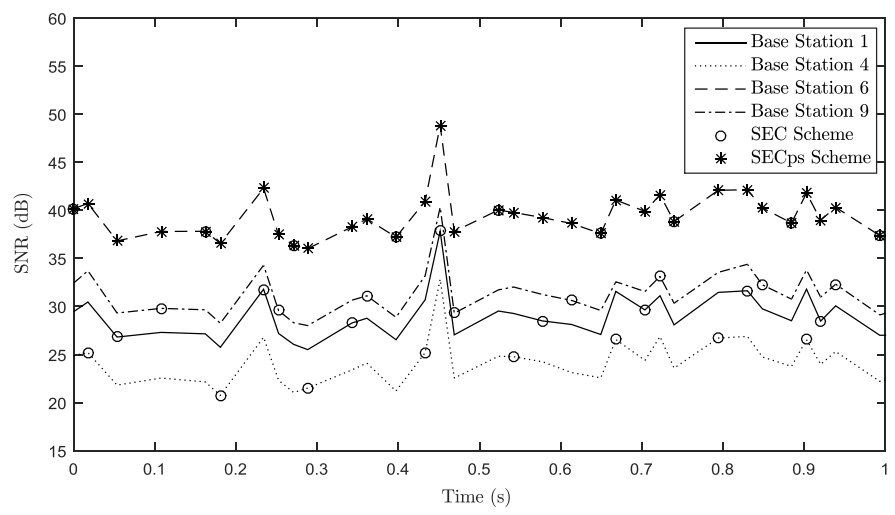

Fig. 10. Excerpt of the SNR time series obtained for scenario 5 comparing four-base station SEC and SECps with the received SNR at base stations 1, 4,6 and 9.

a higher number of signal observations at high SNR levels than the dual-base station configuration. More specifically, as shown in Fig. 11(a), the dual-base station configuration had some output SNR levels which were less than $15 \mathrm{~dB}$ whereas the four-base station configuration had no output SNR less than $15 \mathrm{~dB}$. This result indicates that an additional improvement in the signal quality can be achieved by implementing a four-base station macro-diversity configuration, in which case all SNR drops below $15 \mathrm{~dB}$ level will be eradicated. Again, it was observed that the theoretical PDFs provided an adequate fit to empirical PDFs with the exception of the dual- and four-base station SEC schemes at SNR levels below the switching threshold. Table IV provides a sample of the parameter estimates obtained for both dual- and four-base station SEC and SECps with a medium switching threshold selected for scenario 2 to allow reader to reproduce the theoretical probability densities presented in Fig. 11.

\section{Outage Probability}

The OP is an important performance measure for wireless communication systems. It can be defined as the probability that the instantaneous SNR drops below a minimum threshold required to support acceptable performance, such that

$$
P_{\text {out }}=\operatorname{Pr}\left[\gamma \leq \gamma_{\text {Outage }}\right]=F_{\gamma}\left(\gamma_{\text {Outage }}\right)
$$

where $\gamma_{\text {Outage }}$ is the pre-determined SNR threshold. For the SSC, SEC and SECps schemes, the corresponding OP can be readily obtained by replacing $\gamma$ in (3), (7) and (12) with $\gamma_{\text {Outage. }}$ As an example, Fig. 12 shows the OPs of the four-base station SEC and SECps schemes with group 3 for scenarios 3 and 5 when a medium switching threshold was adopted. For comparison, the OPs of four-base station PSC are also illustrated in Fig. 12. It is clear that the theoretical OPs provided a good fit to the empirical OPs for both the SEC and SECps schemes. Furthermore, it can be seen that the SECps scheme outperformed the SEC scheme for the lower outage threshold levels, particularly below the switching threshold $(30 \mathrm{~dB})$. Finally, it is also worth remarking that, similar to the results presented in Section IV, the four-base station SECps set-up provided almost the same performance as the four-base station PSC configuration.

\section{CONCLUSION}

The potential improvement in signal reliability for outdoor wearable communications at $868 \mathrm{MHz}$ using macro-diversity arrangements based on dual-base station SSC, SEC and SECps 

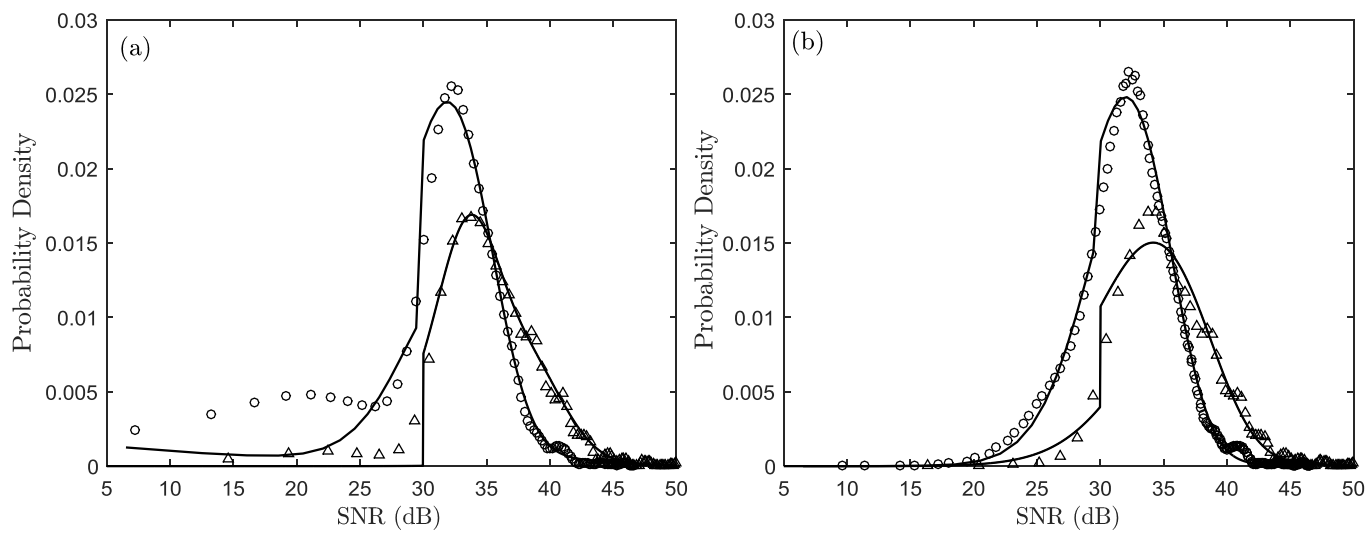

Fig. 11. Empirical (symbols) and theoretical (continuous lines) PDFs for scenario 2 for dual-base station configuration with group 2 (circle symbols) and four-base station configuration with group 4 (triangle symbols) for both (a) SEC and (b) SECps schemes with a medium switching threshold (30 dB).

TABLE IV

Parameter Estimates for Dual-Base Station SEC And SECps with Group 2 (Base Stations 5 and 10) and Four-Base Station SEC and SECps with Group 4 (Base Stations 3, 5, 8, And 10) at the Medium Switching Threshold for Scenario 2.

\begin{tabular}{ccccccccccccc}
\hline \hline & \multicolumn{9}{c}{ Dual-base Station } & \multicolumn{9}{c}{ Four-base Station } \\
& $m_{1}$ & $\overline{\gamma_{1}}$ & $m_{2}$ & $\overline{\gamma_{2}}$ & $m_{1}$ & $\overline{\gamma_{1}}$ & $m_{2}$ & $\overline{\gamma_{2}}$ & $m_{3}$ & $\overline{\gamma_{3}}$ & $m_{4}$ & $\overline{\gamma_{4}}$ \\
\hline SEC & 6.99 & 46.45 & 0.50 & 51.68 & 0.88 & 100.00 & 0.88 & 100.00 & 6.01 & 81.90 & 15.00 & 49.58 \\
SECps & 6.86 & 47.09 & 0.50 & 26.92 & 4.64 & 65.64 & 0.78 & 100.00 & 0.50 & 0.10 & 10.52 & 0.10 \\
\hline \hline
\end{tabular}

and four-base station SEC and SECps has been investigated. The benefit of having more than two signal base stations has been demonstrated by comparing the diversity gains between a range of dual- and four-base station configurations. It was found that for the scenarios considered here, switched diversity systems consisting of a four-base station configuration were less affected by the location of the base stations and thus provided a more stable performance (in terms of signal reliability). Among the three different switched diversity schemes, the SECps scheme provided the best performance for all scenarios and groupings. The results have shown that this type of switched diversity system can provide up to $19.5 \mathrm{~dB}$ diversity gain when using a four-base station arrangement. However, this improvement is not open-ended as it will add extra complexity to the design of the switched diversity system.

The impact of different switching thresholds on the diversity gain, the number of path examinations and the number of path switches of the various combiner outputs have been studied to emphasize the importance of selecting an appropriate switching threshold. It was observed that there exists an optimum switching threshold which maximizes the performance for this type of the wearable system. More specifically, at the optimum switching threshold, the switched diversity system can provide considerable performance with much fewer path examinations when compared to the PSC scheme. It was also found that while a four-base station switched diversity system provides higher diversity gain than a dual-base station switched diversity system, this comes at the cost of having a greater number of path examinations and switches. This result indicates that there exists a trade off between diversity gain (performance) and the number of path examinations and path switches between base stations (energy consumption).

In direct contrast to this, an inappropriate switching threshold may cause not only the substandard performance of wearable systems but also greater energy consumption due to the unnecessary path examinations and needless path switches between base stations. In this case, no additional benefit is obtained using a switched combining based macro-diversity system. Consequently, the appropriate switching threshold must be carefully chosen so that the switched combining based macro-diversity system works as desired. In this study, each scenario was found to have a different optimum switching threshold. Nonetheless, among the three switching threshold levels which were considered, the medium switching threshold $(30 \mathrm{~dB})$ was found to be quite close to the optimum switching threshold for all of the scenarios.

Finally, a statistical analysis of the switched diversity combiner output has been presented under the assumption of independent and non-identically distributed Nakagami- $m$ fading channels. Over all of the measurement scenarios considered in this study, the PDFs of the switched combiner including the SSC, SEC and SECps schemes have been shown to provide an adequate fit to the fading observed at the output of the virtual switched combiner. From the parameter estimates, it was found that each base station experienced non-identical Nakagami- $m$ fading. The parameter estimates also suggest that the fading conditions observed at each base station varied according to the user's movement.

\section{ACKNOWLEDGEMENT}

This work was supported by the U.K. Engineering and Physical Sciences Research Council (EPSRC) under Grant Reference EP/L026074/1. 

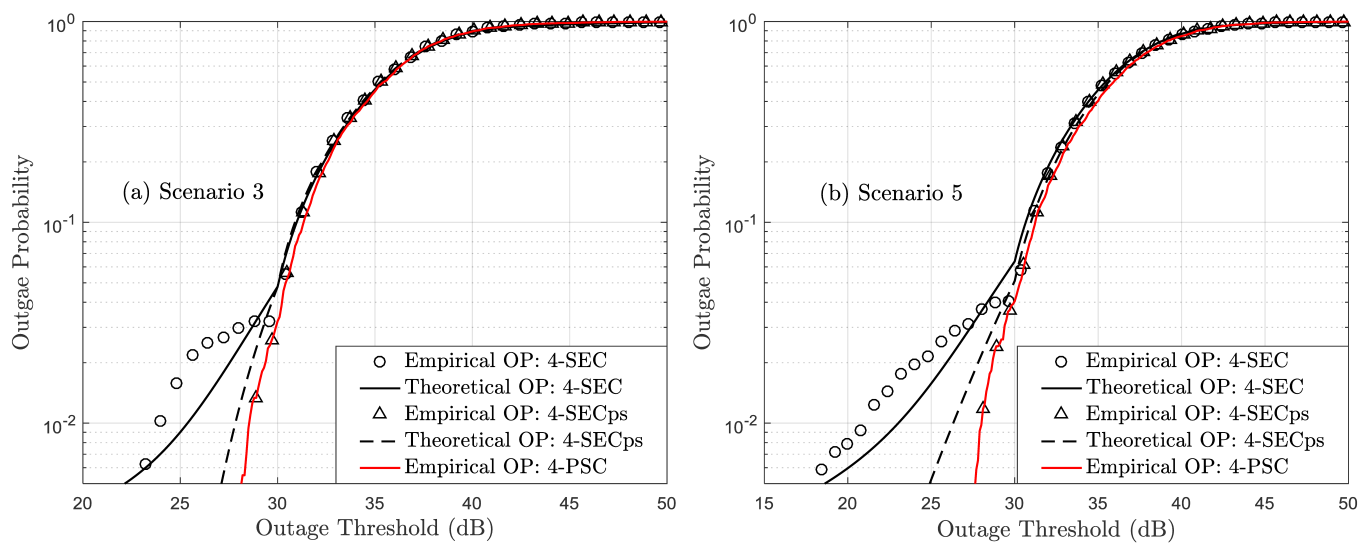

Fig. 12. Empirical and theoretical OPs for four-base station SEC and SECps with group 3 for scenarios 3 and 5 with a medium switching threshold (30 dB) alongside the empirical OP for four-base station PSC. It is worth highlighting that the parameter estimates given in Table III are used for the theoretical OPs.

\section{REFERENCES}

[1] W. C. Jakes, Microwave mobile communications. Wiley-IEEE Press, 1974.

[2] C. B. Dietrich, K. Dietze, J. R. Nealy, and W. L. Stutzman, "Spatial, polarization, and pattern diversity for wireless handheld terminals," IEEE Trans. Antennas Propag., vol. 49, no. 9, pp. 1271-1281, Sep. 2001.

[3] J. Boutros and E. Viterbo, "Signal space diversity: a power- and bandwidth-efficient diversity technique for the Rayleigh fading channel," IEEE Trans. Inf. Theory, vol. 44, no. 4, pp. 1453-1467, Jul. 1998.

[4] G. L. Stüber, Principles of mobile communication, 3rd ed. Springer, 2011.

[5] M. D. Yacoub, Foundations of mobile radio engineering. CRC press, 1993.

[6] M. K. Simon and M.-S. Alouini, Digital communication over fading channels, 2nd ed. New York: Wiley, 2005.

[7] S. K. Yoo and S. L. Cotton, "Improving signal reliability for indoor off-body communications using spatial diversity at the base station," in Proc. IEEE EuCAP, Hague, Netherlands, Apr. 2014, pp. 857-861.

[8] A. J. Ali, S. L. Cotton, and W. G. Scanlon, "Spatial diversity for offbody communications in an indoor populated environment at $5.8 \mathrm{GHz}$," in Proc. IEEE LAPC, Loughborough, UK, Nov. 2009, pp. 641-644.

[9] Q. H. Abbasi, M. M. Khan, A. Alomainy, and Y. Hao, "Diversity antenna techniques for enhanced ultra wideband body-centric communications," in Proc. IEEE APSURSI, Washington, USA, Jul. 2011, pp. 1323-1326.

[10] S. L. Cotton and W. G. Scanlon, "Characterization and modeling of onbody spatial diversity within indoor environments at $868 \mathrm{MHz}$," IEEE Trans. Wireless Commun., vol. 8, no. 1, pp. 176-185, Jan. 2009.

[11] Q. H. Abbasi, M. U. Rehman, H. T. Chattha, K. Qaraqe, A. Alomainy, Y. Hao, and C. Parini, "Ultra wideband antenna diversity characterisation for off-body communications in an indoor environment," IET Microw. Antennas Propag., vol. 8, no. 14, pp. 1161-1169, Nov. 2014.

[12] I. Khan, P. S. Hall, A. A. Serra, A. R. Guraliuc, and P. Nepa, "Diversity performance analysis for on-body communication channels at 2.45 GHz," IEEE Trans. Antennas Propag., vol. 57, no. 4, pp. 956-963, Apr. 2009.

[13] T. Liang and D. B. Smith, "Energy-efficient, reliable wireless body area networks: cooperative diversity switched combining with transmit power control," IET Electron. Lett., vol. 50, no. 22, pp. 1641-1643, Oct. 2014.

[14] D. B. Smith, "Cooperative switched combining for wireless body area networks," in Proc. IEEE PIMRC, Sydney, Australia, Sep. 2012, pp. 2275-2280.

[15] S. L. Cotton, R. D'Errico, and C. Oestges, "A review of radio channel models for body centric communications," Radio Science, vol. 49, no. 6 , pp. 371-388, Jun. 2014.

[16] M. Patel and J. Wang, "Applications, challenges, and prospective in emerging body area networking technologies," IEEE Wireless Commun., vol. 17, no. 1, pp. 80-88, Feb. 2010.

[17] S. L. Cotton, W. G. Scanlon, and A. McKernan, "Improving signal reliability in outdoor body-to-body communications using front and back positioned antenna diversity," in Proc. IEEE EuCAP, Prague, Czech Republic, Mar. 2012, pp. 3393-3396.
[18] S. L. Cotton and W. G. Scanlon, "Measurements, modeling and simulation of the off-body radio channel for the implementation of bodyworn antenna diversity at $868 \mathrm{MHz}$," IEEE Trans. Antennas Propag., vol. 57, no. 12, pp. 3951-3961, Dec. 2009.

[19] H. C. Yang and M.-S. Alouini, "Performance analysis of multibranch switched diversity systems," IEEE Trans. Commun., vol. 51, no. 5, pp. 782-794, May 2003.

[20] R. Rosini and R. D'Errico, "Off-body channel modelling at $2.45 \mathrm{GHz}$ for two different antennas," in Proc. IEEE EuCAP, Prague, Czech, Mar. 2012, pp. 3378-3382.

[21] M. Kim and J. I. Takada, "Characterization of wireless on-body channel under specific action scenarios at sub-GHz bands," IEEE Trans. Antennas Propag., vol. 60, no. 11, pp. 5364-5372, Nov. 2012.

[22] H. Suzuki, "A statistical model for urban radio propogation," IEEE Trans. Commun., vol. 25, no. 7, pp. 673-680, Jul. 1977.

[23] W. R. Braun and I. Dersch, "A physical mobile radio channel model," IEEE Trans. Veh. Technol., vol. 40, no. 2, pp. 472-482, May 1991.

[24] M. Nakagami, "The $m$-distribution: A general formula of intensity distribution of rapid fading," Statistical Method of Radio Propagation, vol. W. C. Hoffman, New York: Pergamon Press, 1960.

[25] A. Abdi and M. Kaveh, "K distribution: an appropriate substitute for Rayleigh-lognormal distribution in fading-shadowing wireless channels," IET Electron. Lett., vol. 34, no. 9, pp. 851-851, Apr. 1998.

[26] I. M. Kostić, "Analytical approach to performance analysis for channel subject to shadowing and fading," IEE Proc. Comm., vol. 152, no. 6, pp. 821-827, Apr. 2005.

[27] Z. Song, K. Zhang, and L. Y. Guan, "Generating correlated nakagami fading signals with arbitrary correlation and fading parameters," in Proc. IEEE ICC, New York, USA, Apr. 2002, pp. 1363-1367.

[28] I. S. Gradshteyn and I. M. Ryzhik, Table of integrals, series, and products, 7th ed. London: Academic Press, 2007.

[29] H. C. Yang and M.-S. Alouini, "Improving the performance of switched diversity with post-examining selection," IEEE Trans. Wireless Commun., vol. 5, no. 1, pp. 67-71, Jan. 2006.

[30] M. Deriche and A. H. Tewfik, "Maximum likelihood estimation of the parameters of discrete fractionally differenced Gaussian noise process," IEEE Trans. Signal Process., vol. 41, no. 10, pp. 2977-2989, Oct. 1993.

[31] D. Crecraft and S. Gergely, Analog Electronics: circuits, systems and signal processing. Butterworth-Heinemann, 2002.

[32] A. Turkmani, A. Arowojolu, P. Jefford, and C. Kellett, "An experimental evaluation of the performance of two-branch space and polarization diversity schemes at $1800 \mathrm{MHz}$," IEEE Trans. Veh. Technol., vol. 44, no. 2, pp. 318-326, May 1995.

[33] D. H. Johnson and S. Sinanovic, Symmetrizing the Kullback-Leibler distance. Rice University Working Paper, 2001.

[34] S. Kullback, Information theory and statistics. Courier Corporation, 1997. 


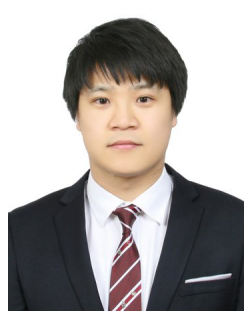

Seong Ki Yoo received the B.Eng. (Hons.) degree in telecommunication systems from the University of Surrey, Guildford, U.K., in 2010, and the M.Sc. degree in communications and signal processing from Imperial College London, London, U.K., in 2012. He is currently pursuing the Ph.D. degree with Queen's University Belfast, Belfast, U.K. His Ph.D. studies have been sponsored by U.K. EPSRC. His research interests are in the areas of fading channel characterization and modeling for wearable communications and diversity in wearable applications.

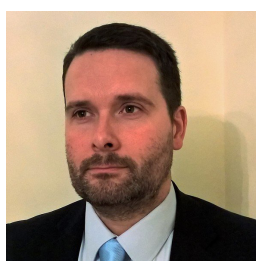

Simon L. Cotton (S'04-M'07-SM'14) received the B.Eng. degree in electronics and software from the University of Ulster, Ulster, U.K., in 2004 and the $\mathrm{Ph} . \mathrm{D}$. degree in electrical and electronic engineering from Queen's University of Belfast, Belfast, U.K., in 2007. He is currently a Reader in wireless communications with the Institute of Electronics, Communications and Information Technology (ECIT), Queen's University Belfast. He is also a Co-founder of and the Chief Technology Officer with ActivWireless Ltd, Belfast, U.K. He has authored and co-authored over 100 publications in major IEEE/IET journals and refereed international conferences, two book chapters, and two patents. Among his research interests are cellular device-to-device, vehicular, and wearable communications. His other research interests include radio channel characterization and modeling and the simulation of wireless channels. Dr. Cotton was awarded the H. A. Wheeler Prize, in July 2010, by the IEEE Antennas and Propagation Society for the best applications journal paper in the IEEE TRANSACTIONS ON ANTENNAS AND PROPAGATION during 2009. In July 2011, he was awarded the Sir George Macfarlane Award from the U.K. Royal Academy of Engineering in recognition of his technical and scientific attainment since graduating from his first degree in engineering.

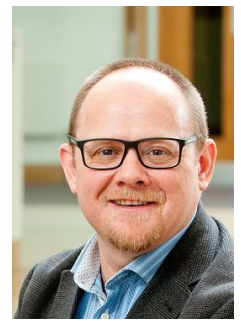

William G. Scanlon (1969) received the B.Eng. degree in electrical engineering and the Ph.D. degree in electronics (specializing in wearable and implanted antennas) from the University of Ulster, U.K., in 1994 and 1997, respectively. He was appointed as Lecturer at the University of Ulster in 1998 , Senior Lecturer and Full Professor at Queen's University of Belfast (U.K.) in 2002 and 2008, respectively. $\mathrm{He}$ is currently Chair of Wireless Communications and Director of the Centre for Wireless Innovation at Queen's and he held a part-time Chair in Short Range Radio at the University of Twente, The Netherlands from 2009 to 2014. Prior to starting his academic career he had 10 years of industrial experience, having worked as a Senior RF Engineer for Nortel Networks, as a Project Engineer with Siemens and as a Lighting Engineer with GECOsram. His current research interests include mobile, personal and bodycentric wireless communications, wearable antennas, RF and microwave propagation, channel modelling and characterization, wireless networking and protocols and wireless networked control systems. He has published more than 230 technical papers in major IEEE/IET journals and in refereed international conferences. He served as keynote speaker for the IEEE Intl Microwave Workshop Series on RF and Wireless Technologies for Biomedical and Healthcare Applications (2014), the NATO Military Communications and Information Systems Conf. (2010), the Intl. Conf. on Bodynets (2010) and the European Workshop on Conformal Antennas (2007). He has been a Series Editor of the IET Book Series on Telecommunications and Networking, he was an inaugural Associate Editor of the IEEE Journal of Translational Engineering in Health and Medicine and he is an Associate Editor for IEEE Antennas and Wireless Propagation Letters. Prof. Scanlon received a Young Scientist award from URSI in 1999, he was a recipient of the 2010 IEEE $\mathrm{H}$ A. Wheeler Prize Paper Award for IEEE Trans. Antennas and Propagation and he delivered the 2012 NATO International Lecture Series on Next Generation Communications. Professor Scanlon is Managing Director and co-founder of ActivWireless Ltd, a Queen's University spin-out company focussed on Real Time Locating Systems and student attendance monitoring using active RFID.

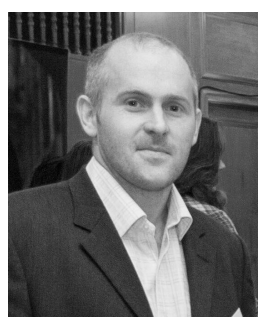

Gareth A. Conway received a BEng Hons. degree in Electronic Systems from the University of Ulster, U.K., in 2004. In 2008, he completed a Ph.D. degree in Electronic Engineering, entitled 'Wearable Antennas for On-Body Wireless Communications' at Queen's University of Belfast, (U.K.). On completion of his doctorate, he spent three years as a commercial research engineer, specializing in antennas and propagation for mobile communication. In 2011, he re-joined QUB to complete an EPSRC Knowledge Transfer Secondment with Toumaz Healthcare Ltd.,undertaking research and development in 'Innovative bodyworn antennas for medical devices'. In 2013 he became a Lecturer in Communications Engineering at ECIT, Queen's University of Belfast. Dr. Conway has authored or co-authored 26 international conference and journal papers. His research interests include antennas, human tissue equivalent materials, wave propagation and computational electromagnetism for wearable and implantable communications. 